\title{
GLOBAL EXISTENCE TO THE CAUCHY PROBLEM FOR HYPERBOLIC CONSERVATION LAWS WITH AN ISOLATED UMBILIC POINT
}

\begin{abstract}
BY
ELISABETTA FELACO (Department of Mathematics, University of Hamburg, Bundesstrasse 55 Hamburg, 20146, Germany),

BRUNO RUBINO (Department of Mathematics, University of L'Aquila, via Vetoio 1, L'Aquila, 67100, Italy),

AND

ROSELLA SAMPALMIERI (Department of Mathematics, University of L'Aquila, via Vetoio 1, L'Aquila, 67100, Italy)

Abstract. In this paper the existence of global weak solutions for a $2 \times 2$ system of non-strictly hyperbolic non-linear conservation laws is established for data in $L^{\infty}$.

The result is proven by means of viscous approximation and application of the compensated compactness method.

The presence of a degeneracy in the hyperbolicity of the system requires a careful analysis of the entropy functions, whose regularity is necessary to obtain an existence result. For this purpose we combine the classical techniques referring to a singular EulerPoisson-Darboux equation with the compensated compactness method.
\end{abstract}

1. Introduction and main result. We consider the following system:

$$
\left\{\begin{array}{l}
u_{t}+f(u, v)_{x}=0, \\
v_{t}+g(u, v)_{x}=0, \\
(u, v)_{\mid t=0}=\left(u_{0}, v_{0}\right),
\end{array}\right.
$$

where $u=u(x, t), v=v(x, t)$ and $(x, t) \in \mathbb{R} \times \mathbb{R}^{+}$.

Using the compact notation $U=(u, v)$ and $F=(f, g)$, we can write the system as

$$
\begin{cases}U_{t}+F(U)_{x} & =0 \\ U_{\mid t=0} & =U_{0}=\left(u_{0}, v_{0}\right)\end{cases}
$$

Received October 4, 2011 and, in revised form, March 27, 2012.

2010 Mathematics Subject Classification. Primary 35L65, 35L80.

E-mail address: elisabetta.felaco@math.uni-hamburg.de

E-mail address: rubino@ing.univaq.it

E-mail address: sampalm@ing.univaq.it 
We assume $U_{0} \in L^{\infty}, F \in C^{1}$, and we denote $D F(U)=\left(\begin{array}{cc}f_{u} & f_{v} \\ g_{u} & g_{v}\end{array}\right)$.

We require that hyperbolicity only fails in one point (umbilic point), that we can identify with the origin, and that the genuine non-linearity only fails on a curve $\gamma$, which will be part of the boundary of our domain.

To assume that the origin is an isolated umbilic point means that the eigenvalues coincide only in $(0,0)$. Such umbilic degeneracy allows a sort of interaction, or nonlinear resonance, between distinct modes and leads to singularities (this phenomenon missing in the strictly hyperbolic case) $(23)$.

The flux function that we consider has the following main feature:

$$
\begin{aligned}
& f(u, v)=\frac{3}{2} u^{2}+\frac{1}{2} v^{2}+\phi(u, v), \\
& g(u, v)=u v+\psi(u, v),
\end{aligned}
$$

where $\phi$ and $\psi$ are smooth functions such that $\phi(u, v)=O\left((|u|+|v|)^{l}\right)$ and $\psi(u, v)=$ $O\left((|u|+|v|)^{l}\right)$, where $l>0$ is a constant to be determined.

We recall that, for a regular function $f, f(x)=O\left(x^{l}\right)$ if and only if there exist two constants $c_{1}>0$ and $c_{2}>0$ such that $c_{1}<\left|\frac{f(x)}{x^{l}}\right|<c_{2}$, in a neighbourhood of $x \rightarrow 0$.

Interest in degenerate hyperbolic equations dates back to the work of Euler who proposed the Euler-Poisson-Darboux equation. This kind of study has been applied to get a solution for a non-linear system of entropic gas dynamics ([6], [7, 11], 18]).

Moreover, non-linear hyperbolic systems with such a kind of degeneracy have found applications in many different areas as multiphase flow in porous media, elasticity, magnetohydrodynamics and oil reservoir simulations ([10, [15, [23, [27, 30]).

This type of umbilic singularity appears naturally in multidimensional systems of conservation laws, as was proven in ([16]).

The local existence problem for such systems is well developed because many techniques from linear equations can be used. However, because of the non-linearity, the solutions of the Cauchy problem develop singularities even with smooth initial data, this being a reflection of the physical phenomena of breaking of weaves and the development of shock weaves. There have been several efforts to understand the Riemann solutions for such a system ([12, 24, 28]). Moreover, two kinds of degeneracy have been classified, each governing a different behaviour of the solution near the umbilic point: parabolic degeneracy and hyperbolic degeneracy (15]).

More literature arises for non-strictly hyperbolic systems with degeneracy along a line (see 19 and the references therein).

We gather inspiration from the ideas that can be found in the papers of Kan ([13], [14]), Chen-Kan ([1], 2]), and Rubino ([22, [21]), all based on the theory of compensated compactness developed by Tartar ([31]), Murat ([20]), Di Perna ([7]) and Serre ([26]) for strictly hyperbolic systems.

The main idea is to prove the existence of uniformly bounded solutions $U^{\varepsilon}$ for the viscous approximating system of (1.2) and then prove that $\left\{U^{\varepsilon}\right\}_{\varepsilon>0}$ converges, for $\varepsilon \rightarrow 0$, to a solution $U$ of (1.2) by reducing the Young measure, associated to $\left\{U^{\varepsilon}\right\}_{\varepsilon>0}$, to a delta function. 
The main problem in our case, when compared to the case of Serre, is that the failure of strict hyperbolicity at the umbilic point causes the partial differential equation governing the entropy functions to have singular coefficients, but regular entropies are needed in order to apply the div-curl lemma and to obtain the commutation relation in the theory of compensated compactness.

We will construct a class of regular entropies as solutions to special Goursat problems in the following way: first using Riemann's method to get an integral representation for entropy functions, and then imposing a special integral condition on the Goursat data in order to cancel the singular part of the Riemann function in such representation.

The first existence theorems of global solutions have been obtained by Kan ([13], 14]) for the case

$$
\begin{aligned}
& f(u, v)=\frac{3}{2} u^{2}+\frac{1}{2} v^{2} \\
& g(u, v)=u v .
\end{aligned}
$$

Here one can explicitly compute the Riemann invariants and so give a complete study of the geometry of the system. In this case it is also possible to explicitly find a family of Riemann functions for the entropy equation and study its regularity properties.

Kan's method was later extended to more general systems, and it is in this potentiality for extensions that its importance relies, since for the system (1.4) itself the compensated compactness method could be carried out in a simpler way ([5], [17]).

Chen and Kan ([1], 2]) generalized Kan's previous result to the quadratic flux function

$$
\begin{aligned}
& f(u, v)=\frac{a}{2} u^{2}+b u v+\frac{1}{2} v^{2}, \\
& g(u, v)=\frac{b}{2} u^{2}+u v
\end{aligned}
$$

with some technical hypothesis on the coefficients $a, b$.

This choice is significant as, from the results in [23, it follows the existence of a nonsingular linear coordinate transformation that transforms every system with quadratic flux in a system with this type of flux.

In this case the methods used by Kan still apply, with some more technical difficulties. Some of these difficulties can be overcome thanks to the second order homogeneity of the flux function, that allows one to carry on the study of the system introducing the variable $\alpha=u / v$.

Frid and Santos ([9]) generalised Kan's work to some particular cases of non-strictly hyperbolic conservation laws of the conjugate type, while Rubino ([21], 22]) generalized it to a system of the form

$$
\begin{aligned}
& f(u, v)=a(u)+b(v), \\
& g(u, v)=u v
\end{aligned}
$$

with $b(v)=k v^{2 n}+o\left(|v|^{2 n}\right)$ and $a(u)=\left(n+\frac{1}{2}\right) u^{2}+o\left(|u|^{2}\right), n \in \mathbb{N}, k>0$.

As we can see, in this last case, the main hypotheses are that the flux function is the same as that of Kan up to second order terms and that there are no higher order terms that include the product of both the state variables $u$ and $v$.

The main difficulty here, with respect to Kan's paper, is that it is not possible to explicitly solve the Riemann function problem so that the author prefers to prove that, near the umbilic point, the Riemann function has the same regularity as the one derived 
by Kan so that, again, a suitable condition on the Goursat data gives a family of regular entropies.

Our goal is to generalize the previous results, first of all by also adding higher order terms to the flux function in the second equation, and mainly by including the case of flux functions where the two state variables are non-separated, at least in the terms of order greater than 2 .

The paper contains two main results. The first one is the following Lemma 1.1 that is a fundamental technical result concerning the existence of regular entropies for the perturbed system. This regularity was taken for granted in the previous works, while here we present a detailed proof through the partial results of Section 4, Section 5 and Section 6.

LEMMA 1.1 (Existence of regular entropies). Under hypothesis (1.3), with $l>7$, there exists a choice of Goursat data for which the solutions of the entropy equation associated to system (1.1) are bounded near the origin up to the second order derivative.

The second result we present is a result on the Cauchy problem (1.1), establishing the existence of a global weak solution:

TheOrem 1.2 (Global existence). Consider system (1.1) with

$$
\begin{aligned}
& f(u, v)=\frac{3}{2} u^{2}+\frac{1}{2} v^{2}+\phi(u, v), \\
& g(u, v)=u v+\psi(u, v),
\end{aligned}
$$

where $\phi$ and $\psi$ satisfy the hypotheses of Lemma 1.1 .

Assume (1.1) to be a symmetric system where strict hyperbolicity fails only in $(0,0)$. Also assume $u_{0}, v_{0}, \partial_{x} u_{0}, \partial_{x} v_{0} \in L^{\infty}$ and $\left(u_{0}, v_{0}\right) \in I^{+}=\left\{(u, v) \mid v+\phi_{v} \geq 0\right\}$. Then the sequence $\left(u^{\varepsilon}, v^{\varepsilon}\right)$ of solutions of the viscous approximation of (1.1),

$$
\left\{\begin{array}{l}
u_{t}+f(u, v)_{x}=\varepsilon u_{x x}, \\
v_{t}+g(u, v)_{x}=\varepsilon v_{x x}, \\
(u, v)_{\mid t=0}=\left(u_{0}, v_{0}\right),
\end{array}\right.
$$

converges strongly in $L_{l o c}^{p}, p<+\infty$, to a weak solution $(u, v)$ of (1.1).

For our purposes we actually need (1.1) to be hyperbolic, with strict hyperbolicity failing only in the origin; we assume symmetry to simplify notation.

The result $l>7$ appears of course to be non-optimal, but is the best possible to obtain with this technique and in our general setting. The condition arises in Section 5 when proving the regularity of solutions of the entropy equation (see Theorem 5.5).

We would like to emphasize that the results in the previous sections, among which is the basic Lemma 5.2, hold true for the more weak hypothesis $l>3$.

This work is organised as follows.

In Section 2, we start our analysis of the mathematical problem, studying the geometry of the rarefaction wave curves in order to construct a set of Riemann invariants for system (1.1) and to analyse their properties.

In Section 3 we recall some known results on parabolic systems that guarantee the existence of $L^{\infty}$ uniformly bounded solutions for the viscous system. 
In Section 4 we introduce the entropy equation and Serre's method (see [26]) to construct a family of entropy solutions bounded up to the second derivatives in the state variables. This is non-trivial, as the equation governing the entropy functions has coefficients that are singular at the umbilic point.

In Section 5 we will give our main result: following [13, [14] and [21, and using the Riemann representation method of solutions, we will find a set of suitable conditions in order to get regular entropies.

Finally, in Section 6, we will establish the compensated compactness framework. We will set up the Young measure and the commutation relation, and then use the entropies constructed in Section 5 to reduce the Young measure to a point mass.

We add, for the reader's convenience, an Appendix with some of the results in [13, as it is actually unpublished.

2. Riemann invariants and their properties. As already pointed out in the introduction, we assume $D F(U)$ to be symmetric. This will make it easier to write conditions to have an isolated umbilic point and to find a convex entropy for the system.

So we assume, now and for the sequel,

$$
f_{v}=g_{u} .
$$

The eigenvalues of system (1.1) are

$$
\lambda_{ \pm}=\frac{\left(g_{v}+f_{u}\right) \pm \sqrt{G(u, v)}}{2},
$$

while the eigenvectors are

$$
r_{ \pm}=\left(\begin{array}{c}
2 f_{v} \\
\left(g_{v}-f_{u}\right) \pm \sqrt{G(u, v)}
\end{array}\right)
$$

where $G(u, v)=\left(g_{v}-f_{u}\right)^{2}+4\left(f_{v}\right)^{2}$.

System (1.1) is strictly hyperbolic as long as $\lambda_{ \pm}$are real valued and satisfy $\lambda_{-}<\lambda_{+}$, so, in order to have an isolated umbilic point in the origin, we need $G(u, v)$ to verify $G(u, v)=0 \Longleftrightarrow(u, v)=(0,0)$, that gives us the condition

$$
\left\{\begin{array}{l}
\left(g_{v}-f_{u}\right)(u, v)=0 \\
f_{v}(u, v)=0 .
\end{array} \Longleftrightarrow(u, v)=(0,0)\right.
$$

2.1. Construction of Riemann invariants. Let us recall that the Riemann invariants $\left(\omega_{-}, \omega_{+}\right)$for (1.1) satisfy $\nabla \omega_{ \pm} \times r_{\mp}=0$ and that the integral curves $R_{ \pm}$of $r_{ \pm}$in the state space are called the first and the second rarefaction wave curve, respectively.

The Riemann invariants, by definition, are constant along $R_{ \pm}$, and these curves satisfy the dynamical system

$$
\left\{\begin{array}{l}
\dot{u}=2 f_{v}(u, v) \\
\dot{v}=\left(g_{v}-f_{u}\right)(u, v) \pm \sqrt{G(u, v)} .
\end{array}\right.
$$

In the general case, it is not always possible to find an exact solution for this equation, so we will need to study the geometry of the rarefaction waves curves, which is also 
essential to obtain a $L^{\infty}$ a priori estimate for the viscous approximate solutions by means of the invariant regions technique.

We consider the sets

$$
I^{+}:=\left\{(u, v) \subseteq \mathbb{R}^{2} \mid f_{v}(u, v) \geq 0\right\} \quad \text { and } \quad I^{-}:=\left\{(u, v) \subseteq \mathbb{R}^{2} \mid f_{v}(u, v) \leq 0\right\}
$$

separated by the curve $v=\gamma(u)$, implicitly defined by the equation $f_{v}(u, v)=0$, once we ask $f_{v v} \neq 0$. Let $I=I^{+} \cup I^{-}$.

REMARK 2.1. We want $\gamma$ to be monotone (where the constant case $\gamma(u)=0$ is the unperturbed case). To this aim we have to add the hypothesis that $\gamma^{\prime}$ does not change sign. Since in the unperturbed case we have $f_{v v}=1$, it seems natural to assume

$$
f_{v v}>0,
$$

so $\gamma$ is non-decreasing if $f_{u v} \leq 0$ and non-increasing if $f_{u v} \geq 0$. The construction of the Riemann invariants is possible in both cases; we will restrict our study to the case

$$
f_{u v} \geq 0
$$

when it is easier to write some of the technical conditions on the second order derivatives of $f$ and $g$.

Now we state the qualitative properties of $R_{ \pm}$in $I^{+}$, but it is completely similar to deal with $R_{ \pm}$in the complementary domain $I^{-}$.

LEMMA 2.2. In our setting of hypotheses the following properties hold:

(i) the part of the $\gamma$ curve that is in the fourth (second) quadrant is an $R_{+}\left(R_{-}\right)$ curve;

(ii) the $R_{+}\left(R_{-}\right)$curves are in one to one correspondence with the points of the part of the $\gamma$ curve that is in the second (fourth) quadrant;

(iii) every $R_{+}\left(R_{-}\right)$which does not coincide with the curve $\gamma$ keeps getting further from the curve $\gamma$ as u goes to $+\infty(-\infty)$.

Finally for (1.1) we can construct Riemann invariants $\left(\omega_{-}, \omega_{+}\right)$so that

$$
\omega_{-} \leq 0 \leq \omega_{+} .
$$

Proof. By the uniqueness of solutions for system (2.3) and the previous construction we only have to prove (iii); we will prove the $R_{+}$curves case.

In our set of hypotheses we have:

$$
\frac{d v}{d u}-\gamma^{\prime}(u)=\frac{\left(g_{u}-f_{v}\right)+\sqrt{G}}{2 f_{v}}+\frac{f_{u v}}{f_{v v}} \geq 0
$$

so that the distance between the $R_{+}$curves and $\gamma$ increases on the right.

Now, since $\omega_{ \pm}$is constant along every $R_{\mp}$, if we prescribe $\omega_{ \pm}$on $\gamma$ as follows: for every $(u, v)$ such that $v=\gamma(u)$ we assign

$$
\begin{aligned}
& \omega_{-}(u, v)= \begin{cases}u & \text { if } u<0, \\
0 & \text { if } u \geq 0,\end{cases} \\
& \omega_{+}(u, v)= \begin{cases}0 & \text { if } u \leq 0, \\
u & \text { if } u>0 .\end{cases}
\end{aligned}
$$


Then for every $(u, v) \in I^{+}$we have well defined Riemann invariants such that $\omega_{-}(u, v) \leq 0 \leq \omega_{+}(u, v)$, as shown in Fig. 1)

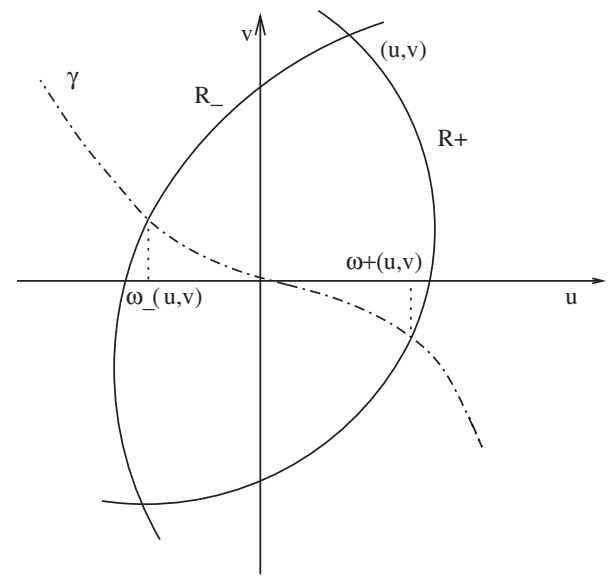

FIG. 1. Construction of Riemann invariants

Now it is possible to define the mapping

$$
\begin{aligned}
& T: I^{+} \subseteq \mathbb{R}^{2} \quad \longrightarrow \quad J=T(I) \subseteq \mathbb{R}^{2}, \\
& (u, v) \quad \longrightarrow \quad\left(\omega_{-}(u, v), \omega_{+}(u, v)\right) \text {, }
\end{aligned}
$$

which is a well defined change of coordinates from $I^{+}=\left\{(u, v) \subseteq \mathbb{R}^{2} \mid f_{v}(u, v) \geq 0\right\}$ to $J=\left\{\left(\omega_{-}, \omega_{+}\right) \subseteq \mathbb{R}^{2} \mid \omega_{-} \leq 0 \leq \omega_{+}\right\}$.

However, as the strict hyperbolicity fails at the umbilic point, this transformation is not regular at the origin.

From the geometry of the rarefaction wave curves we also have:

COROLlary 2.3. The Riemann invariants constructed as above satisfy

$$
\begin{aligned}
& \frac{1}{f_{v}} \frac{\partial \omega_{-}}{\partial v}<0, \\
& \frac{1}{f_{v}} \frac{\partial \omega_{+}}{\partial v}>0
\end{aligned}
$$

for all $(u, v) \in I$ such that $f_{v}(u, v) \neq 0$.

Finally we remark on another property of the Riemann invariants that will be useful in Section 3 to construct invariant regions:

Lemma 2.4. For $(u, v) \neq(0,0)$, if

$$
L_{ \pm}(u, v):=-\left(f_{v}\right)^{2} \partial_{u} z_{\mp}+f_{v v}\left(z_{\mp}\right)^{2}-f_{v} z_{\mp} \partial_{v} z_{\mp}+f_{v} f_{u v} z_{\mp} \geq 0,
$$

then the following holds:

$$
\left\{\begin{array}{l}
\nabla^{2} \omega_{+}\left(r_{-}, r_{-}\right) \geq 0 \\
\nabla^{2} \omega_{-}\left(r_{+}, r_{+}\right) \leq 0 .
\end{array}\right.
$$

That is, $\pm \omega_{ \pm}$are quasiconvex in the sense of [3]. 
Proof. First of all recall that $\nabla \omega_{ \pm} \times r_{\mp}=0$ and that

$$
r_{ \pm}=\left(\begin{array}{c}
f_{v} \\
z_{ \pm}
\end{array}\right)
$$

where $z_{ \pm}:=\frac{\left(g_{v}-f_{u}\right) \pm \sqrt{G}}{2}$, so we have

$$
f_{v}\left(\partial_{u} \omega_{ \pm}\right)+z_{\mp}\left(\partial_{v} \omega_{ \pm}\right)=0 .
$$

Differentiating (2.4) with respect to $u$ we get

$$
f_{u v}\left(\partial_{u} \omega_{ \pm}\right)+f_{v}\left(\partial_{u}^{2} \omega_{ \pm}\right)+\left(\partial_{u} z_{\mp}\right)\left(\partial_{v} \omega_{ \pm}\right)+z_{\mp}\left(\partial_{v}^{2} \omega_{ \pm}\right)=0,
$$

from which we have

$$
f_{v}\left(\partial_{u}^{2} \omega_{ \pm}\right)=-f_{u v}\left(\partial_{u} \omega_{ \pm}\right)-\left(\partial_{u} z_{\mp}\right)\left(\partial_{v} \omega_{ \pm}\right)-z_{\mp}\left(\partial_{v}^{2} \omega_{ \pm}\right),
$$

and, multiplying for $\left(\partial_{v} \omega_{ \pm}\right)^{2} / f_{v}$,

$$
\left(\partial_{u}^{2} \omega_{ \pm}\right)\left(\partial_{v} \omega_{ \pm}\right)^{2}=-\frac{f_{u v}}{f_{v}}\left(\partial_{u} \omega_{ \pm}\right)\left(\partial_{v} \omega_{ \pm}\right)^{2}-\frac{\partial_{u} z_{\mp}}{f_{v}}\left(\partial_{v} \omega_{ \pm}\right)^{3}-\frac{z_{\mp}}{f_{v}}\left(\partial_{v} \omega_{ \pm}\right)^{2}\left(\partial_{u v}^{2} \omega_{ \pm}\right) .
$$

By (2.4), we can write $\partial_{v} \omega_{ \pm}=-\frac{f_{v}}{z_{\mp}}\left(\partial_{u} \omega_{ \pm}\right)$and $\partial_{u} \omega_{ \pm}=-\frac{z_{\mp}}{f_{v}}\left(\partial_{v} \omega_{ \pm}\right)$so that we finally get

$$
\left(\partial_{u}^{2} \omega_{ \pm}\right)\left(\partial_{v} \omega_{ \pm}\right)^{2}-\left(\partial_{u} \omega_{ \pm}\right)\left(\partial_{v} \omega_{ \pm}\right)\left(\partial_{u v}^{2} \omega_{ \pm}\right)=-\frac{\partial_{u} z_{\mp}}{f_{v}}\left(\partial_{v} \omega_{ \pm}\right)^{3}+\frac{f_{u v}}{f_{v}^{2}} z_{\mp}\left(\partial_{v} \omega_{ \pm}\right)^{3} .
$$

In a similar way, differentiating with respect to $v$ we get

$$
\left(\partial_{v}^{2} \omega_{ \pm}\right)\left(\partial_{u} \omega_{ \pm}\right)^{2}-\left(\partial_{u} \omega_{ \pm}\right)\left(\partial_{v} \omega_{ \pm}\right)\left(\partial_{u v}^{2} \omega_{ \pm}\right)=-\frac{\partial_{v} z_{\mp}}{\left(f_{v}\right)^{2}} z_{\mp}\left(\partial_{v} \omega_{ \pm}\right)^{3}+\frac{f_{v v}}{\left(f_{v}\right)^{3}}\left(z_{\mp}\right)^{2}\left(\partial_{v} \omega_{ \pm}\right)^{3} .
$$

Now, adding (2.5) and (2.6) , we find

$$
\nabla^{2} \omega_{ \pm}\left(r_{\mp}, r_{\mp}\right)=\left(\frac{\partial_{v} \omega_{ \pm}}{f_{v}}\right)^{3}\left\{-\left(f_{v}\right)^{2} \partial_{u} z_{\mp}+f_{v v}\left(z_{\mp}\right)^{2}-f_{v} z_{\mp} \partial_{v} z_{\mp}+f_{v} f_{u v} z_{\mp}\right\} .
$$

So, thanks to Corollary 2.3, we only need the hypothesis

$$
L_{ \pm}(u, v):=-\left(f_{v}\right)^{2} \partial_{u} z_{\mp}+f_{v v}\left(z_{\mp}\right)^{2}-f_{v} z_{\mp} \partial_{v} z_{\mp}+f_{v} f_{u v} z_{\mp} \geq 0 .
$$

2.2. Genuine non-linearity. The following analysis of the genuine non-linearity properties for system (1.1) will be used when studying the reduction of Young measure in Section 6.

Let's recall that a system of conservation laws is said to be genuinely non-linear in a domain $A$ if

$$
\nabla \lambda_{ \pm}(u, v) \times r_{ \pm}(u, v) \neq 0
$$

for all $(u, v) \in A$.

In the case of system (1.1) the genuine non-linearity fails for all $(u, v)$ such that

$$
\begin{aligned}
& \left\{\frac{\left(g_{v}+f_{u}\right)_{u}}{2} \pm \frac{\left(g_{v}-f_{u}\right)\left(g_{v}-f_{u}\right)_{u}+4 f_{v} f_{u v}}{2 \sqrt{G}}\right\} 2 f_{v} \\
& +\left\{\frac{\left(g_{v}+f_{u}\right)_{v}}{2} \pm \frac{\left(g_{v}-f_{u}\right)\left(g_{v}-f_{u}\right)_{v}+4 f_{v} f_{v v}}{2 \sqrt{G}}\right\}\left(\left(g_{v}-f_{u}\right) \pm \sqrt{G}\right)=0 .
\end{aligned}
$$


First of all let's observe that on $v=\gamma(u)$ (2.7) becomes

$$
\left(\left(g_{v}-f_{u}\right)+\left|g_{v}-f_{u}\right|\right)\left\{\frac{\left(g_{v}+f_{u}\right)_{v}}{2}+\frac{\left(g_{v}-f_{u}\right)\left(g_{v}-f_{u}\right)_{v}}{2\left|g_{v}-f_{u}\right|}\right\}=0
$$

that is verified outside the origin for all $(u, v)$ if $\left(g_{v}-f_{u}\right)(u, v)<0$, and

$$
\left(\left(g_{v}-f_{u}\right)-\left|g_{v}-f_{u}\right|\right)\left\{\frac{\left(g_{v}+f_{u}\right)_{v}}{2}-\frac{\left(g_{v}-f_{u}\right)\left(g_{v}-f_{u}\right)_{v}}{2\left|g_{v}-f_{u}\right|}\right\}=0
$$

that is verified outside the origin for all $(u, v)$ such that $\left(g_{v}-f_{u}\right)(u, v)>0$.

As one of the main characteristics that we require system (1.1) to verify is that the genuine non-linearity fails only on the boundary of our domain $I^{+}$, we now look for conditions that assure

$$
\nabla \lambda_{ \pm}(u, v) \times r_{ \pm}(u, v)=0 \text { iff } v=\gamma(u) .
$$

From (2.7) and using $f_{v v}=g_{u v}$, it follows that:

Proposition 2.5. For system (1.1) the genuine non-linearity fails only on $\gamma$ if we choose $f$ and $g$ such that

$$
\begin{array}{r}
6 f_{v} f_{v v}\left(\left(g_{v}-f_{u}\right)+\sqrt{G}\right)-2 f_{v} f_{u u}\left(\left(g_{v}-f_{u}\right)-\sqrt{G}\right) \\
+12 f_{v}^{2} f_{u v}+g_{v v}\left(\left(g_{v}-f_{u}\right)-\sqrt{G}\right)^{2}=0
\end{array}
$$

if and only if $v=\gamma_{+}:=\left\{\begin{array}{ll}f_{v} & =0, \\ \left(g_{v}-f_{u}\right) & \leq 0\end{array}\right.$ and

$$
\begin{array}{r}
-6 f_{v} f_{v v}\left(\left(g_{v}-f_{u}\right)-\sqrt{G}\right)+2 f_{v} f_{u u}\left(\left(g_{v}-f_{u}\right)+\sqrt{G}\right) \\
-12 f_{v}^{2} f_{u v}-g_{v v}\left(\left(g_{v}-f_{u}\right)-\sqrt{G}\right)^{2}=0
\end{array}
$$

if and only if $v=\gamma_{-}:= \begin{cases}f_{v} & =0 \\ \left(g_{v}-f_{u}\right) & \geq 0\end{cases}$

Notice that in the $\left(\omega_{-}, \omega_{+}\right)$-plane $\gamma_{-}$corresponds to $\omega_{-}=0$ and that $\gamma_{+}$corresponds to $\omega_{+}=0$.

Finally, it can be proven that $\partial_{\omega_{-}} \lambda_{-}$and $\partial_{\omega_{+}} \lambda_{+}$remain non-zero on the axes except possibly at the umbilic point $u=v=0=\omega_{-}=\omega_{+}$. This result will be useful in the reduction of the Young measure.

LEMma 2.6. If $\left(g_{v}-f_{u}\right)_{u} \neq 0$ on $\gamma_{+}$and $\gamma_{-}$then the following holds:

(i) $\frac{\partial \lambda_{-}}{\partial \omega_{-}} \neq 0$ on $\left\{\omega_{-}<0, \omega_{+}=0\right\}$,

(ii) $\frac{\partial \lambda_{+}}{\partial \omega_{+}} \neq 0$ on $\left\{\omega_{-}=0, \omega_{+}>0\right\}$.

REMARK 2.7 (Perturbation of Kan's quadratic model).

(i) Our goal is to prove the existence of global solutions for system (1.1) under the hypothesis (1.3):

$$
\begin{aligned}
& f(u, v)=\frac{3}{2} u^{2}+\frac{1}{2} v^{2}+\phi(u, v), \\
& g(u, v)=u v+\psi(u, v),
\end{aligned}
$$


where $\phi(u, v)=O\left((|u|+|v|)^{l}\right)$ and $\psi(u, v)=O\left((|u|+|v|)^{l}\right)$. This means that we want to consider flux functions that are higher order perturbations of the quadratic flux function corresponding to the case studied by Kan. Moreover, we are interested in analysing the situation only in a small neighbourhood of the origin, as elsewhere the known results on strictly hyperbolic systems hold.

(ii) We would like to translate the hypotheses we have stated until now in terms of $\phi$ and $\psi$. We are looking for $\phi(u, v)=O\left((|u|+|v|)^{l}\right)$ and $\psi(u, v)=O\left((|u|+|v|)^{l}\right)$, such that

$$
\begin{gathered}
\phi_{v} \quad=\psi_{u}, \\
\phi_{u v} \geq 0 \\
1+\phi_{v v}>0 \\
\begin{cases}\left(-2 u+\psi_{v}(u, v)-\phi_{u}(u, v)\right) & =0 \\
v+\phi_{v}(u, v)=0 & \Longleftrightarrow(u, v)=(0,0)\end{cases}
\end{gathered}
$$

and we restrict our analysis to the domain

$$
I^{+}=\left\{(u, v) \subseteq \mathbb{R}^{2} \mid v+\phi_{v}(u, v) \geq 0\right\} .
$$

(iii) We also notice that Lemma 2.4 holds under hypothesis (1.3) if we restrict our analysis in a neighbourhood of the origin. This means that, under our hypotheses, $\pm \omega_{ \pm}$are quasiconvex in the sense of $([3])$. Moreover, we remark that under hypothesis (1.3) Lemma 2.6 holds, as $\left(g_{v}-f_{u}\right)_{u}=-2+\left(\psi_{v}-\phi_{u}\right)_{u}$ is strictly negative in a neighbourhood of the origin.

As for the genuine non-linearity, we can easily prove that, under hypothesis (1.3), it fails only on the boundary $\gamma$ of $I^{+}$.

(iv) We can consider as an example the case

$$
\begin{aligned}
& \phi(u, v)=a u^{3} v+b u^{2} v^{2}+c u v^{3}, \\
& \psi(u, v)=\frac{a}{4} u^{4}+\frac{2}{3} b u^{3} v+\frac{3}{2} c u^{2} v^{2}
\end{aligned}
$$

with $a \geq 0, c \geq 0$ and $b^{2} \leq \frac{3}{4} c$.

Here we have the symmetry by construction and every hypotheses holds in a small neighbourhood of the origin. The conditions on the parameters $a, b$ and $c$ are needed to construct the Riemann invariants as in Section 2.1.

3. Viscous approximation. In this section we establish the existence of $L^{\infty}$ bounded solutions for the following viscous approximation to system (1.1):

$$
\left\{\begin{array}{l}
u_{t}+f(u, v)_{x}=\varepsilon u_{x x}, \\
v_{t}+g(u, v)_{x}=\varepsilon v_{x x} \\
(u, v)_{\mid t=0}=\left(u_{0}, v_{0}\right)
\end{array}\right.
$$

where $\varepsilon>0$.

We also establish that, if the initial data are in the closed subset $I^{+} \subset \mathbb{R}^{2}$, then every solution of (3.1) remains in it.

In order to show that (3.1) has a global solution in time, we first prove the existence of invariant regions for (3.1) in order to have a $L^{\infty}$ uniform bound for the solutions. A 
fixed point argument will complete the proof. In the sequel we will refer to the classical results from [3] and [29].

Theorem 3.1 (See Theorem 4.4, p. 379 [3]). Let $\Sigma=\left\{(u, v) \mid s_{-}(u, v) \leq 0\right\} \cap\{(u, v) \mid$ $\left.s_{+}(u, v) \leq 0\right\}$, where $s_{ \pm}$are smooth functions defined on a convex set.

Assume that for all $t>0$ and all $(\bar{u}, \bar{v}) \in \partial \Sigma$, the following holds:

(i) $\nabla s_{ \pm}$is a left eigenvector of $D F(\bar{u}, \bar{v})$;

(ii) $s_{ \pm}$is quasiconvex at $(\bar{u}, \bar{v})$.

Then $\Sigma$ is invariant for (3.1) for every $\varepsilon>0$.

We also recall that a smooth function $S: \mathbb{R}^{n} \longrightarrow \mathbb{R}$ is quasiconvex in the sense of [3] at $\zeta \in \mathbb{R}^{n}$ if

$$
\nabla S \times \zeta=0 \Rightarrow \nabla^{2} S(\zeta, \zeta) \geq 0 .
$$

In our case we pick $s_{-}=-c-\omega_{-}$and $s_{+}=\omega_{+}-c$, where $c>0$ is an arbitrary constant and $s_{ \pm}: I=I^{+} \cup I^{-} \rightarrow \mathbb{R}$.

Then (i) is satisfied by the definition of Riemann invariants, so we only need to prove (ii).

As we know that $\nabla \omega_{ \pm} \times r_{\mp}=0$, (ii) holds under the hypothesis of Lemma 2.4, and thanks to the previous result, we have that:

\section{Proposition 3.2.}

$$
\Sigma_{c}=\left\{(u, v) \in I \mid \omega_{-}+c \geq 0\right\} \cap\left\{(u, v) \in I \mid \omega_{+}-c \leq 0\right\}
$$

is a family of invariant regions for system 3.1, where $c>0, \Sigma_{c}$ is strictly increasing in $c$ and it spans the whole phase plane when $c \longrightarrow+\infty$.

We can conclude that, if the data $\left(u_{0}, v_{0}\right) \in L^{\infty}$, then there exists a constant $c>0$ such that the solution $\left(u^{\varepsilon}, v^{\varepsilon}\right)$ of (3.1), when it exists, is in $\Sigma_{c}$ for all $(x, t) \in \mathbb{R} \times \mathbb{R}^{+}$and $\varepsilon>0$.

Next we assume $\left(u_{0}, v_{0}\right) \in L^{\infty}$ in (3.1) and we prove the global existence of a solution by a fixed point argument (see [29]).

THEOREm 3.3. If $\left(u_{0}, v_{0}\right) \in L^{\infty}$, then system (3.1) has a unique solution, global in time: $\left(u^{\varepsilon}, v^{\varepsilon}\right) \in C((0, \infty) ; C(\mathbb{R}))$.

Finally, in the last part of this section we want to prove that if we take our initial data $\left(u_{0}, v_{0}\right) \in I^{+}=\left\{(u, v) \subseteq \mathbb{R}^{2} \mid f_{v}(u, v) \geq 0\right\}$, then the solution $\left(u^{\varepsilon}, v^{\varepsilon}\right)$ remains in $I^{+}$, for every $\varepsilon>0$.

Using a positive solution theorem from the theory of parabolic equations (see, for example, [8]), we can prove:

Proposition 3.4. If $\left(u_{0}, v_{0}\right) \in I^{+} \cap L^{\infty}$, then every solution $\left(u^{\varepsilon}, v^{\varepsilon}\right)$ of (3.1) belongs to $I^{+}$for all $(x, t) \in \mathbb{R} \times \mathbb{R}^{+}$.

Proof. We know from classic results that for a parabolic system such as (3.1), under reasonable assumptions, positive initial data lead to positive solutions. We apply this result to the functions $z:=v-\gamma(u)$ and $z_{0}:=v_{0}-\gamma\left(u_{0}\right)$ and show that $z_{0} \geq 0 \Rightarrow z \geq$ 0 . 
Corollary 3.5. Under the previous assumptions we have, for all $(x, t) \in \mathbb{R} \times[0, \infty)$,

$$
w^{*}-\lim _{\varepsilon \rightarrow 0^{+}}\left(u^{\varepsilon}, v^{\varepsilon}\right) \in I^{+} .
$$

4. Serre's method to construct regular entropies. In order to apply the theory of compensated compactness as developed by Serre in [26] we need to prove the existence of a family of regular entropies for system (1.1). We want to construct four canonical types of entropies that will be called entropies of type East, West, North and South, following the standard notation. Each one of these entropies vanishes on a half plane and is obtained by solving a Goursat problem for the partial differential equation governing the entropy in the characteristic variables. The theory in [26] shows how to construct such entropies as long as the system is strictly hyperbolic; however, in our case, the entropies of type East and type South are, in general, singular at the umbilic point, as their support contains the origin. So we will use an integral representation for these types of entropies using Riemann's method and we will set additional conditions on the Goursat data to obtain regular entropies in each of the four cases. Since in our general setting it is not possible to explicitly compute the Riemann function for the entropy equation, we will prove that, at least in a neighbourhood of the origin, our Riemann function has the same behaviour as that of the case studied by Kan, and we will use such a result to derive the necessary integral condition.

Definition 4.1. The couple $(\eta, q)$ is an entropy-entropy flux pair for (1.1) if, for any classical solution $U=(u, v)$ of (1.1), we have

$$
\eta(u, v)_{t}+q(u, v)_{x}=0 .
$$

This yields to the compatibility condition $\nabla q=\nabla \eta D F(U)$.

Eliminating $q$ we get a second order partial differential equation in $\eta$ :

$$
f_{v}\left(\eta_{v v}-\eta_{u u}\right)+\left(f_{u}-g_{v}\right) \eta_{u v}=0 .
$$

REMARK 4.2. It is easy to check that $\eta^{*}=u^{2}+v^{2}$ is a convex solution of (4.1). More generally, $\eta^{k}=k_{1}\left(u^{2}+v^{2}\right)+k_{2}$ is an entropy for the system for every $k_{1}, k_{2} \in \mathbb{R}$. These simple entropies will be useful in Section 6 .

Equation (4.1) in $\left(\omega_{-}, \omega_{+}\right)$coordinates reads as

$$
\frac{\partial^{2} \eta}{\partial \omega_{+} \partial \omega_{-}}+\frac{1}{\lambda_{+}-\lambda_{-}}\left(\frac{\partial \lambda_{+}}{\partial \omega_{-}} \frac{\partial \eta}{\partial \omega_{+}}-\frac{\partial \lambda_{-}}{\partial \omega_{+}} \frac{\partial \eta}{\partial \omega_{-}}\right)=0
$$

which we are interested in solving only when $\omega_{-} \leq 0 \leq \omega_{+}$.

Now we consider the Goursat problem associated to (4.2), that is, the problem of finding a solution of (4.2) when its value is known on two incident characteristics:

$$
\left\{\begin{aligned}
\frac{\partial^{2} \eta}{\partial \omega_{+} \partial \omega_{-}}+\frac{1}{\lambda_{+}-\lambda_{-}}\left(\frac{\partial \lambda_{+}}{\partial \omega_{-}} \frac{\partial \eta}{\partial \omega_{+}}-\frac{\partial \lambda_{-}}{\partial \omega_{+}} \frac{\partial \eta}{\partial \omega_{-}}\right) & =0 \\
\eta\left(\omega_{-}, \omega_{+}^{*}\right) & =\theta_{-}\left(\omega_{-}\right), \\
\eta\left(\omega_{-}^{*}, \omega_{+}\right) & =\theta_{+}\left(\omega_{+}\right),
\end{aligned}\right.
$$

where $\left(\omega_{-}^{*}, \omega_{+}^{*}\right)$ are fixed constants such that $\omega_{-}^{*} \leq 0 \leq \omega_{+}^{*}$ and $\theta_{-}, \theta_{+}$are given smooth functions such that $\theta_{-}\left(\omega_{-}^{*}\right)=\theta_{+}\left(\omega_{+}^{*}\right)$. 
Notice that the coefficients of the first order terms in (4.2) are singular at the umbilic point.

Our main goal in this section is to find some conditions on the Goursat data in order to obtain solutions to (4.3) whose derivatives, up to the second order, are bounded in

$$
J^{*}:=\left\{\left(\omega_{-}, \omega_{+}\right) \mid \omega_{-}^{*} \leq \omega_{-} \leq 0 \leq \omega_{+} \leq \omega_{+}^{*}\right\} .
$$

We must now recall four canonical classes of entropies:

DeFinition 4.3. We call entropy of type East with limit $\omega_{-}^{*}$ a solution to problem (4.3) when

$$
\begin{aligned}
& \omega_{+}^{*}=0, \\
& \omega_{-}^{*}<0, \\
& \theta_{+} \equiv 0, \\
& \theta_{-}=0 \quad \text { if } \omega_{-} \leq \omega_{-}^{*} .
\end{aligned}
$$

In a similar way we can define entropy of type West with limit $\omega_{-}^{*}$ and entropy of type South and entropy of type North with limit $\omega_{+}^{*}$.

As the support of entropies of types West and North does not contain the umbilic point, we can use the known results to have regular solutions.

In the next section we will analyse in detail the case of entropies of type East, the case of South type entropies being similar.

\section{Main result.}

5.1. The Riemann function for the entropy equation. To study the regularity of entropy of type East, we will make use of an integral representation obtained via the Riemann function associated to equation (4.2).

For classical results we refer to chapter 5 of [4].

We want to study the problem

$$
\left\{\begin{aligned}
\frac{\partial^{2} \eta}{\partial \omega_{+} \partial \omega_{-}}+\frac{1}{\lambda_{+}-\lambda_{-}}\left(\frac{\partial \lambda_{+}}{\partial \omega_{-}} \frac{\partial \eta}{\partial \omega_{+}}-\frac{\partial \lambda_{-}}{\partial \omega_{+}} \frac{\partial \eta}{\partial \omega_{-}}\right) & =0 \\
\eta\left(\omega_{-}, 0\right) & \equiv \theta_{-}\left(\omega_{-}\right) \\
\eta\left(\omega_{-}^{*}, \omega_{+}\right) & =0
\end{aligned}\right.
$$

with $\theta_{-}\left(\omega_{-}\right)=0$ if $\omega_{-} \leq \omega_{-}^{*}$.

Our first step will be to derive an integral representation for the solution $\eta$ of (5.1).

Let

$$
\mathcal{R}=\mathcal{R}\left(t, s ; \omega_{-}, \omega_{+}\right)
$$

be the Riemann function for the entropy equation (4.2). We know that $\mathcal{R}$ contains all the information about the general solution of the problem and is defined as the solution to the characteristic boundary value problem for the adjoint equation of (4.2) with respect to the first couple of variables:

$$
\frac{\partial^{2} \mathcal{R}}{\partial s \partial t}+\left(\frac{\partial_{\omega_{+}} \lambda_{-}}{\lambda_{+}-\lambda_{-}} \mathcal{R}\right)_{t}-\left(\frac{\partial_{\omega_{-}} \lambda_{+}}{\lambda_{+}-\lambda_{-}} \mathcal{R}\right)_{s}=0
$$


with special boundary data:

$$
\begin{gathered}
\mathcal{R}\left(t, s ; \omega_{-}, s\right)=\exp \left\{\int_{\omega_{-}}^{t} \frac{\partial_{\omega_{-}} \lambda_{+}}{\lambda_{+}-\lambda_{-}}(x, s) d x\right\} \\
\mathcal{R}\left(t, s ; t, \omega_{+}\right)=\exp \left\{-\int_{\omega_{+}}^{s} \frac{\partial_{\omega_{+}} \lambda_{-}}{\lambda_{+}-\lambda_{-}}(t, y) d y\right\} .
\end{gathered}
$$

It is well known (again see [4]) that the solution to (5.1), at least in regions where $\eta$ is $C^{2}$, can be expressed in integral form in terms of $\mathcal{R}$ and $\theta_{-}$as follows:

$$
\eta\left(\omega_{-}, \omega_{+}\right)=\int_{\omega_{-}^{*}}^{\omega_{-}} \mathcal{R}\left(t, 0 ; \omega_{-}, \omega_{+}\right)\left(\theta_{-}^{\prime}(t)-\frac{\partial_{\omega_{-}} \lambda_{+}(t, 0)}{\lambda_{+}(t, 0)-\lambda_{-}(t, 0)} \theta_{-}(t)\right) d t .
$$

The idea is to analyse the existence and the singular behaviour of $\mathcal{R}$ and then use representation (5.2) to show how to choose a general class of data $\theta_{-}$to cancel those singularities.

To this end we first show that, near the umbilic point, $\mathcal{R}$ has the same behaviour as $\mathcal{R}_{0}$, where $\mathcal{R}_{0}$ is the Riemann function for the entropy equation associated to the unperturbed system (see the Appendix).

In what follows we will use the subscript 0 when referring to the unperturbed case. First notice that equation (4.2) can be written as

$$
\frac{\partial^{2} \eta}{\partial \omega_{+} \partial \omega_{-}}+\frac{1}{2\left(\left(\omega_{+}\right)_{0}-\left(\omega_{-}\right)_{0}\right)}\left(\frac{\partial \eta}{\partial \omega_{+}}-\frac{\partial \eta}{\partial \omega_{-}}\right)=\rho\left(\omega_{-}, \omega_{+}\right) \frac{\partial \eta}{\partial \omega_{+}}+\sigma\left(\omega_{-}, \omega_{+}\right) \frac{\partial \eta}{\partial \omega_{-}}
$$

where

$$
\rho\left(\omega_{-}, \omega_{+}\right)=\frac{1}{2\left(\left(\omega_{+}\right)_{0}-\left(\omega_{-}\right)_{0}\right)}-\frac{\partial_{\omega_{-}} \lambda_{+}}{\lambda_{+}-\lambda_{-}}
$$

and

$$
\sigma\left(\omega_{-}, \omega_{+}\right)=-\left(\frac{1}{2\left(\left(\omega_{+}\right)_{0}-\left(\omega_{-}\right)_{0}\right)}-\frac{\partial_{\omega_{+}} \lambda_{-}}{\lambda_{+}-\lambda_{-}}\right) .
$$

If $\rho \equiv \sigma \equiv 0$, equation (5.3) is the entropy equation for the unperturbed case.

In order to obtain the main result of this section we need the following lemmas.

First we observe that, as we have one degree of freedom when choosing the Riemann invariants, it is possible to choose a Riemann invariant system of coordinates $\left(\omega_{-}, \omega_{+}\right)$ for which $\partial u / \partial \omega_{ \pm}$are forced to be constant, while the derivatives $\partial v / \partial \omega_{ \pm}$contain all the non-linearity. This choice is consistent with the results of Kan for the unperturbed system. More precisely, the following holds:

LEMma 5.1. There exists a Riemann invariant system of coordinates for system (1.1) as constructed in Lemma 2.2, for which we have

$$
\begin{aligned}
\frac{\partial u}{\partial \omega_{\mp}} & =\frac{1}{2}, \\
\frac{\partial v}{\partial \omega_{\mp}} & =\frac{1}{2} \frac{\left(g_{v}-f_{u}\right) \mp \sqrt{G}}{2 f_{v}} .
\end{aligned}
$$


Proof. Let $T$ be the map that transforms the state variables into the Riemann invariants, $J_{T}$ its Jacobian and $k=\operatorname{det} J_{T}$.

For every $(u, v) \neq(0,0)$, using $J_{T^{-1}}=\left(J_{T}\right)^{-1}$, we get

$$
\frac{\partial \omega_{+}}{\partial v}=k \frac{\partial u}{\partial \omega_{-}} \text {and }-\frac{\partial \omega_{+}}{\partial u}=k \frac{\partial v}{\partial \omega_{-}} .
$$

If we use the relation $\nabla \omega_{ \pm} \times r_{\mp}=0$ we have

$$
\frac{\partial v}{\partial \omega_{\mp}}=\frac{\left(r_{\mp}\right)_{2}}{\left(r_{\mp}\right)_{1}} \frac{\partial u}{\partial \omega_{\mp}} .
$$

As

$$
\frac{\left(r_{\mp}\right)_{2}}{\left(r_{\mp}\right)_{1}}=\frac{\left(g_{v}-f_{u}\right) \mp \sqrt{G}}{2 f_{v}},
$$

we are free to take $\frac{\partial u}{\partial \omega_{\mp}}=\frac{1}{2}$, which gives the result.

With this we can now prove:

LEMmA 5.2. If condition (1.3) holds, that is, if

$$
\begin{aligned}
& f(u, v)=\frac{3}{2} u^{2}+\frac{1}{2} v^{2}+\phi(u, v), \\
& g(u, v)=u v+\psi(u, v),
\end{aligned}
$$

where $\phi(u, v)=O\left((|u|+|v|)^{l}\right)$ and $\psi(u, v)=O\left((|u|+|v|)^{l}\right)$, and if $l>3$, then

$$
\lim _{\left|\omega_{+}-\omega_{-}\right| \longrightarrow 0} \rho\left(\omega_{-}, \omega_{+}\right)=0
$$

and

$$
\lim _{\left|\omega_{+}-\omega_{-}\right| \longrightarrow 0} \sigma\left(\omega_{-}, \omega_{+}\right)=0 .
$$

Proof. We will prove only $\lim _{\left|\omega_{+}-\omega_{-}\right| \longrightarrow 0} \rho\left(\omega_{-}, \omega_{+}\right)=0$, as the other case is similar. First we want to compute

$$
\begin{aligned}
& \frac{\partial \lambda_{+}}{\partial \omega_{-}}=\frac{\partial \lambda_{+}}{\partial u} \frac{\partial u}{\partial \omega_{-}}+\frac{\partial \lambda_{+}}{\partial v} \frac{\partial v}{\partial \omega_{-}} \\
& =\frac{\partial u}{\partial \omega_{-}}\left(\frac{\partial \lambda_{+}}{\partial u}-\frac{\left(r_{-}\right)_{2}}{\left(r_{-}\right)_{1}} \frac{\partial \lambda_{+}}{\partial v}\right) .
\end{aligned}
$$

By the result of the previous lemma, we only have to compute

$$
\begin{gathered}
\frac{\partial \lambda_{+}}{\partial u}-\frac{\left(r_{-}\right)_{2}}{\left(r_{-}\right)_{1}} \frac{\partial \lambda_{+}}{\partial v} \\
=\left(\frac{\left(g_{v}+f_{u}\right)_{u}}{2}+\frac{\left(g_{v}-f_{u}\right)\left(g_{v}-f_{u}\right)_{u}+4 f_{v} f_{u v}}{2 \sqrt{G}}\right) \\
+\frac{\left(g_{v}-f_{u}\right)-\sqrt{G}}{2 f_{v}}\left(\frac{\left(g_{v}+f_{u}\right)_{v}}{2}+\frac{\left(g_{v}-f_{u}\right)\left(g_{v}-f_{u}\right)_{v}+4 f_{v} f_{v v}}{2 \sqrt{G}}\right) .
\end{gathered}
$$

Using hypothesis (1.3) we get

$$
\begin{gathered}
\frac{\partial \lambda_{+}}{\partial u}-\frac{\left(r_{-}\right)_{2}}{\left(r_{-}\right)_{1}} \frac{\partial \lambda_{+}}{\partial v} \\
=1+\frac{\left(\psi_{v}+\phi_{u}\right)_{u}}{2}-\frac{u\left(\psi_{v}-\phi_{u}\right)_{u}}{\sqrt{G}}+\frac{\left(\psi_{v}-\phi_{u}\right)\left(\psi_{v}-\phi_{u}\right)_{u}}{2 \sqrt{G}}
\end{gathered}
$$


Finally,

$$
\begin{gathered}
-\frac{2\left(v+\phi_{v}\right) \phi_{u v}}{\sqrt{G}}+\frac{\left(-2 u+\psi_{v}-\phi_{u}\right) \phi_{u v}}{2\left(v+\phi_{v}\right)}-\frac{\left(\psi_{v}+\phi_{u}\right)_{v} \sqrt{G}}{4\left(v+\phi_{v}\right)} \\
+\frac{\left(-2 u+\psi_{v}-\phi_{u}\right)^{2}\left(\psi_{v}-\phi_{u}\right)_{v}}{4\left(v+\phi_{v}\right) \sqrt{G}}+\frac{\left(-2 u+\psi_{v}-\phi_{u}\right) \phi_{v v}}{\sqrt{G}}-\phi_{v v} .
\end{gathered}
$$

$$
\begin{gathered}
\frac{\partial \lambda_{+}}{\partial \omega_{-}} \\
=\left\{1+\frac{\left(\psi_{v}+\phi_{u}\right)_{u}}{2}-\frac{u\left(\psi_{v}-\phi_{u}\right)_{u}}{\sqrt{G}}+\frac{\left(\psi_{v}-\phi_{u}\right)\left(\psi_{v}-\phi_{u}\right)_{u}}{2 \sqrt{G}}\right. \\
-\frac{2\left(v+\phi_{v}\right) \phi_{u v}}{\sqrt{G}}+\frac{\left(-2 u+\psi_{v}-\phi_{u}\right) \phi_{u v}}{2\left(v+\phi_{v}\right)}-\frac{\left(\psi_{v}+\phi_{u}\right)_{v} \sqrt{G}}{4\left(v+\phi_{v}\right)} \\
\left.+\frac{\left(-2 u+\psi_{v}-\phi_{u}\right)^{2}\left(\psi_{v}-\phi_{u}\right)_{v}}{4\left(v+\phi_{v}\right) \sqrt{G}}+\frac{\left(-2 u+\psi_{v}-\phi_{u}\right) \phi_{v v}}{\sqrt{G}}-\phi_{v v}\right\} \frac{1}{2} .
\end{gathered}
$$

Recalling that $\sqrt{G_{0}}:=\left(\omega_{+}\right)_{0}-\left(\omega_{-}\right)_{0}=2 \sqrt{u^{2}+v^{2}}, \lambda_{+}-\lambda_{-}=\sqrt{G}$, and that, under hypothesis (1.3), we can assume

$$
\sqrt{G}=\sqrt{G_{0}}+O\left((|u|+|v|)^{l}\right)
$$

and

we have

$$
\frac{1}{\sqrt{G}}=\frac{1}{\sqrt{G_{0}}}\left(1+O\left((|u|+|v|)^{l-1}\right)\right)
$$

$$
\begin{gathered}
\rho\left(\omega_{-}, \omega_{+}\right) \\
=\frac{1}{2 \sqrt{G_{0}}}\left\{1-\left(1+O\left((|u|+|v|)^{l-1}\right)\right)\left(1+\frac{\left(\psi_{v}+\phi_{u}\right)_{u}}{2}\right.\right. \\
-\frac{u\left(\psi_{v}-\phi_{u}\right)_{u}}{\sqrt{G}}+\frac{\left(\psi_{v}-\phi_{u}\right)\left(\psi_{v}-\phi_{u}\right)_{u}}{2 \sqrt{G}}-\frac{2\left(v+\phi_{v}\right) \phi_{u v}}{\sqrt{G}} \\
+\frac{\left(-2 u+\psi_{v}-\phi_{u}\right) \phi_{u v}}{2\left(v+\phi_{v}\right)}-\frac{\left(\psi_{v}+\phi_{u}\right)_{v} \sqrt{G}}{4\left(v+\phi_{v}\right)}+\frac{\left(-2 u+\psi_{v}-\phi_{u}\right)^{2}\left(\psi_{v}-\phi_{u}\right)_{v}}{4\left(v+\phi_{v}\right) \sqrt{G}} \\
\left.\left.+\frac{\left(-2 u+\psi_{v}-\phi_{u}\right) \phi_{v v}}{\sqrt{G}}-\phi_{v v}\right)\right\} .
\end{gathered}
$$

Now, by (1.3),

as $l>3$.

$$
\begin{gathered}
\lim _{\left|\omega_{+}-\omega_{-}\right| \longrightarrow 0} \rho\left(\omega_{-}, \omega_{+}\right) \\
=\lim _{(u, v) \longrightarrow(0,0)} \frac{O\left((|u|+|v|)^{l-2}\right)}{\sqrt{u^{2}+v^{2}}}=0,
\end{gathered}
$$

REMARK 5.3. In the previous proposition we cannot allow $l=3$.

If we consider the simple case $\phi=a u^{3}$ and $\psi=b v^{3}$, using polar coordinates, the final limit in the previous proof becomes

$$
\begin{aligned}
& \lim _{(u, v) \longrightarrow(0,0)}-6\left(\frac{a u}{\sqrt{u^{2}+v^{2}}}+\frac{(a+2 b) u^{2}+b v^{2}}{u^{2}+v^{2}}\right) \\
& =\lim _{r \longrightarrow 0}-6\left(a \cos \theta+(a+2 b) \cos ^{2} \theta+b \sin ^{2} \theta\right),
\end{aligned}
$$


that is equal to 0 for every $\theta$ only for specific values of $a$ and $b$.

This proves that the result $l>3$ is optimal within our method.

Now, given $\varepsilon>0$ and $\mathcal{Q}_{\varepsilon}:=(-\varepsilon, 0) \times(0, \varepsilon)$, we can introduce the space

$$
\mathcal{V}:=\left\{F \in C^{2}\left(\mathcal{Q}_{\varepsilon}^{2}, \mathbb{R}\right) \mid\|F\|_{\mathcal{V}}<+\infty\right\}
$$

with the norm

$$
\|F\|_{\mathcal{V}}=\max _{0 \leq|i+j| \leq 2}\left\{\sup _{\mathcal{Q}_{\varepsilon}^{2}}\left|\partial_{\omega_{-}}^{i} \partial_{\omega_{+}}^{j} F\left(t, s ; \omega_{-}, \omega_{+}\right)\right|\left|s-\omega_{-}\right|^{\gamma}|s-t|^{\beta}\left|\omega_{+}-\omega_{-}\right|^{\alpha}\right\},
$$

where $\alpha, \beta$ and $\gamma$ are positive constants to be determined.

REMARK 5.4. $\mathcal{V}$ is a complete vector space, as it is a Frechet space with respect to the separate family of seminorms

$$
\|F\|_{\mathcal{K}_{\delta}}=\sup _{\mathcal{K}_{\delta}^{2}}\|F\|_{\mathcal{V}}
$$

for every $F \in \mathcal{V}$, where $\mathcal{K}_{\delta}=[-\varepsilon+\delta,-\delta] \times[\delta, \varepsilon-\delta], \delta>0$.

The weight in the norm $\|\cdot\|_{\mathcal{V}}$ has been chosen in a polynomial form so that it is possible to balance eventually arising singularities with $|\rho(\cdot, \cdot)|$ and $|\sigma(\cdot, \cdot)|$, that we can control thanks to the previous lemma.

With the results in Lemma 5.2 we can state:

THEOREM 5.5. Let $\mathcal{R}$ be the Riemann function for the entropy equation (4.2) and $\mathcal{R}_{0}$ the Riemann function for the entropy equation associated to the unperturbed system. Under hypothesis (1.3), we have

$$
\lim _{\left|\omega_{+}-\omega_{-}\right| \longrightarrow 0}\left\|\mathcal{R}-\mathcal{R}_{0}\right\|_{\mathcal{V}}=0
$$

if $\alpha>\frac{3}{2}, \beta \geq \frac{5}{2}, \gamma \geq \frac{7}{2}$ and $l>7$.

Proof. Recall from [4 that the Riemann function in any case verifies, with respect to the last two variables, the equation to which it is associated.

So, in particular, $\mathcal{R}$ verifies (4.2).

But, if we consider in (5.3) the right hand side as a function of the independent variables, we can consider $\mathcal{R}_{0}$ as the Riemann function associated to (5.3).

Of course, the representation formula of the solution via the Riemann function in this way becomes an implicit representation formula. In particular, for $\mathcal{R}$ we obtain

$$
\begin{aligned}
\mathcal{R}\left(t, s ; \omega_{-}, \omega_{+}\right) & =\mathcal{R}_{0}\left(t, s ; \omega_{-}, \omega_{+}\right) \\
& +\int_{s}^{\omega_{+}} \mathcal{R}_{0}\left(t, y ; \omega_{-}, \omega_{+}\right)\left(\frac{\partial \mathcal{R}(t, s ; t, y)}{\partial y}+\frac{\mathcal{R}(t, s ; t, y)}{2(y-t)}\right) d y \\
+ & \int_{t}^{\omega_{-}} \mathcal{R}_{0}\left(x, s ; \omega_{-}, \omega_{+}\right)\left(\frac{\partial \mathcal{R}(t, s ; x, s)}{\partial x}+\frac{\mathcal{R}(t, s ; x, s)}{2(x-s)}\right) d x \\
+ & \int_{t}^{\omega_{-}} \int_{s}^{\omega_{+}} \mathcal{R}_{0}\left(x, y ; \omega_{-}, \omega_{+}\right)\left(\rho(x, y) \frac{\partial \mathcal{R}(t, s ; x, y)}{\partial y}\right. \\
& \left.+\sigma(x, y) \frac{\partial \mathcal{R}(t, s ; x, y)}{\partial x}\right) d x d y .
\end{aligned}
$$

We define the operator $\mathcal{T}: \mathcal{V} \longrightarrow \mathcal{V}$ as

$$
\mathcal{T}(\mathcal{R})\left(t, s ; \omega_{-}, \omega_{+}\right)=\mathcal{R}_{0}\left(t, s ; \omega_{-}, \omega_{+}\right)
$$




$$
\begin{aligned}
& +\int_{s}^{\omega_{+}} \mathcal{R}_{0}\left(t, y ; \omega_{-}, \omega_{+}\right) \mathcal{R}(t, s ; t, y) \sigma(t, y) d y+\int_{t}^{\omega_{-}} \mathcal{R}_{0}\left(x, s ; \omega_{-}, \omega_{+}\right) \mathcal{R}(t, s ; x, s) \rho(x, s) d x \\
& \quad+\int_{t}^{\omega_{-}} \int_{s}^{\omega_{+}} \mathcal{R}_{0}\left(x, y ; \omega_{-}, \omega_{+}\right)\left(\rho(x, y) \frac{\partial \mathcal{R}(t, s ; x, y)}{\partial y}+\sigma(x, y) \frac{\partial \mathcal{R}(t, s ; x, y)}{\partial x}\right) d x d y .
\end{aligned}
$$

Our final aim is to prove that $\mathcal{T}$ is a contraction on $\mathcal{V}$. We will only prove that for every $\mathcal{R} \in \mathcal{V}$ also $\mathcal{T}(\mathcal{R}) \in \mathcal{V}$. (The second step in proving that $\mathcal{T}$ is a contraction is similar, just with more complex notation.)

For every $\mathcal{R} \in \mathcal{V}$ we have to show that the expressions

$$
\sup _{\mathcal{Q}_{\varepsilon}^{2}}\left|\partial_{\omega_{-}}^{i} \partial_{\omega_{+}}^{j} \mathcal{T}(\mathcal{R})\left(t, s ; \omega_{-}, \omega_{+}\right)\right|\left|s-\omega_{-}\right|^{\gamma}|s-t|^{\beta}\left|\omega_{+}-\omega_{-}\right|^{\alpha}
$$

are bounded for every $i, j$ such that $0 \leq i+j \leq 2$.

The main problem is represented by the singularities in $\mathcal{R}_{0}$ and in its derivatives near the origin that are of the following kind (see the Appendix for more details): $\ln (1-z)$, $\frac{1}{1-z}$ and $\frac{1}{(1-z)^{2}}$.

Therefore we present the computation for the case involving the worst term, i.e. the one that requires the higher values of $l$.

As an example we consider the expression containing $\partial_{\omega_{-}}^{2} \mathcal{R}_{0}\left(t, s ; \omega_{-}, \omega_{+}\right)$, so we look for the right conditions on $\alpha, \beta, \gamma$ and $l$ to have

$$
\sup _{\mathcal{Q}_{\varepsilon}^{2}}\left|\partial_{\omega_{-}}^{2} \mathcal{T}(\mathcal{R})\left(t, s ; \omega_{-}, \omega_{+}\right)\right|\left|s-\omega_{-}\right|^{\gamma}|s-t|^{\beta}\left|\omega_{+}-\omega_{-}\right|^{\alpha}<+\infty .
$$

From the formula for $\mathcal{T}(\mathcal{R})\left(t, s ; \omega_{-}, \omega_{+}\right)$, we have

$$
\begin{gathered}
\partial_{\omega_{-}} \mathcal{T}(\mathcal{R})\left(t, s ; \omega_{-}, \omega_{+}\right) \\
=\partial_{\omega_{-}} \mathcal{R}_{0}\left(t, s ; \omega_{-}, \omega_{+}\right) \\
+\int_{s}^{\omega_{+}} \partial_{\omega_{-}} \mathcal{R}_{0}\left(t, y ; \omega_{-}, \omega_{+}\right) \mathcal{R}(t, s ; t, y) \sigma(t, y) d y \\
+\mathcal{R}_{0}\left(\omega_{-}, s ; \omega_{-}, \omega_{+}\right) \mathcal{R}\left(t, s ; \omega_{-}, s\right) \rho\left(\omega_{-}, s\right) \\
+\int_{t}^{\omega_{-}} \partial_{\omega_{-}} \mathcal{R}_{0}\left(x, s ; \omega_{-}, \omega_{+}\right) \mathcal{R}(t, s ; x, s) \rho(x, s) d x \\
+\int_{s}^{\omega_{+}} \mathcal{R}_{0}\left(\omega_{-}, y ; \omega_{-}, \omega_{+}\right)\left(\rho\left(\omega_{-}, y\right) \frac{\partial \mathcal{R}\left(t, s ; \omega_{-}, y\right)}{\partial y}+\sigma\left(\omega_{-}, y\right) \frac{\partial \mathcal{R}\left(t, s ; \omega_{-}, y\right)}{\partial \omega_{-}}\right) d y \\
+\int_{s}^{\omega_{+}} \int_{t}^{\omega_{-}} \partial_{\omega_{-}} \mathcal{R}_{0}\left(x, y ; \omega_{-}, \omega_{+}\right)\left(\rho(x, y) \frac{\partial \mathcal{R}(t, s ; x, y)}{\partial y}+\sigma(x, y) \frac{\partial \mathcal{R}(t, s ; x, y)}{\partial x}\right) d x d y .
\end{gathered}
$$

Using this we can compute

$$
\begin{gathered}
\left|\partial_{\omega_{-}}^{2} \mathcal{T}(\mathcal{R})\left(t, s ; \omega_{-}, \omega_{+}\right)\right| \\
\leq\left|\partial_{\omega_{-}}^{2} \mathcal{R}_{0}\left(t, s ; \omega_{-}, \omega_{+}\right)\right| \\
+\int_{s}^{\omega_{+}}\left|\partial_{\omega_{-}}^{2} \mathcal{R}_{0}\left(t, y ; \omega_{-}, \omega_{+}\right)\right||\mathcal{R}(t, s ; t, y)||\sigma(t, y)| d y \\
+\mid \partial_{\omega_{-}}\left(\left(\mathcal{R}_{0}\left(\omega_{-}, s ; \omega_{-}, \omega_{+}\right) \mathcal{R}\left(t, s ; \omega_{-}, s\right) \rho\left(\omega_{-}, s\right)\right) \mid\right. \\
+\left|\partial_{\omega_{-}} \mathcal{R}_{0}\left(\omega_{-}, s ; \omega_{-}, \omega_{+}\right) \mathcal{R}\left(t, s ; \omega_{-}, s\right) \rho\left(\omega_{-}, s\right)\right|
\end{gathered}
$$




$$
\begin{gathered}
+\int_{t}^{\omega_{-}}\left|\partial_{\omega_{-}}^{2} \mathcal{R}_{0}\left(x, s ; \omega_{-}, \omega_{+}\right)\right||\mathcal{R}(t, s ; x, s)||\rho(x, s)| d x \\
+\int_{s}^{\omega_{+}}\left|\partial_{\omega_{-}}\left(\mathcal{R}_{0}\left(\omega_{-}, y ; \omega_{-}, \omega_{+}\right)\left(\rho\left(\omega_{-}, y\right) \frac{\partial \mathcal{R}\left(t, s ; \omega_{-}, y\right)}{\partial y}+\sigma\left(\omega_{-}, y\right) \frac{\partial \mathcal{R}\left(t, s ; \omega_{-}, y\right)}{\partial \omega_{-}}\right)\right)\right| d x \\
+\int_{s}^{\omega_{+}}\left|\partial_{\omega_{-}} \mathcal{R}_{0}\left(\omega_{-}, y ; \omega_{-}, \omega_{+}\right)\right|\left|\left(\rho\left(\omega_{-}, y\right) \frac{\partial \mathcal{R}\left(t, s ; \omega_{-}, y\right)}{\partial y}+\sigma\left(\omega_{-}, y\right) \frac{\partial \mathcal{R}\left(t, s ; \omega_{-}, y\right)}{\partial \omega_{-}}\right)\right| d x \\
+\int_{s}^{\omega_{+}} \int_{t}^{\omega_{-}}\left|\partial_{\omega_{-}}^{2} \mathcal{R}_{0}\left(x, y ; \omega_{-}, \omega_{+}\right)\right|\left|\left(\rho(x, y) \frac{\partial \mathcal{R}(t, s ; x, y)}{\partial y}+\sigma(x, y) \frac{\partial \mathcal{R}(t, s ; x, y)}{\partial x}\right)\right| d x d y .
\end{gathered}
$$

To reach our aim we need to multiply both sides of the inequality for $\left|s-\omega_{-}\right|^{\gamma} \mid s-$ $\left.t\right|^{\beta}\left|\omega_{+}-\omega_{-}\right|^{\alpha}$, take the $\sup _{\mathcal{Q}_{\varepsilon}^{2}}$ and finally show that every term on the right hand side is finite, at least in a neighbourhood of the origin.

As an example we will analyse the term that generate the stronger conditions on $\alpha$ and $l$, i.e. the second one:

$$
\int_{s}^{\omega_{+}}\left|\partial_{\omega_{-}}^{2} \mathcal{R}_{0}\left(t, y ; \omega_{-}, \omega_{+}\right)\right||\mathcal{R}(t, s ; t, y) \| \sigma(t, y)| d y .
$$

So we need to prove

$$
\sup _{\mathcal{Q}_{\varepsilon}^{2}}\left|s-\omega_{-}\right|^{\gamma}|s-t|^{\beta}\left|\omega_{+}-\omega_{-}\right|^{\alpha} \int_{s}^{\omega_{+}}\left|\partial_{\omega_{-}}^{2} \mathcal{R}_{0}\left(t, y ; \omega_{-}, \omega_{+}\right)\right||\mathcal{R}(t, s ; t, y)||\sigma(t, y)| d y<+\infty .
$$

First we write

$$
\begin{aligned}
\sup _{\mathcal{Q}_{\varepsilon}^{2}}\left|s-\omega_{-}\right|^{\gamma}|s-t|^{\beta}\left|\omega_{+}-\omega_{-}\right|^{\alpha} \int_{s}^{\omega_{+}}\left|\partial_{\omega_{-}}^{2} \mathcal{R}_{0}\left(t, y ; \omega_{-}, \omega_{+}\right)\right||\mathcal{R}(t, s ; t, y)||\sigma(t, y)| d y \\
=\sup _{\mathcal{Q}_{\varepsilon}^{2}} \int_{s}^{\omega_{+}}\left|\partial_{\omega_{-}}^{2} \mathcal{R}_{0}\left(t, y ; \omega_{-}, \omega_{+}\right)\right|\left(|\mathcal{R}(t, s ; t, y)||s-t|^{\gamma}|s-t|^{\beta}|y-t|^{\alpha}\right) \\
\cdot\left(\frac{s-\omega_{-}}{s-t}\right)^{\gamma}\left(\frac{\omega_{+}-\omega_{-}}{y-t}\right)^{\alpha}|\sigma(t, y)| d y \\
<\sup _{\mathcal{Q}_{\varepsilon}^{2}} \int_{s}^{\omega_{+}}\left|\partial_{\omega_{-}}^{2} \mathcal{R}_{0}\left(t, y ; \omega_{-}, \omega_{+}\right)\right|\left(\frac{s-\omega_{-}}{s-t}\right)^{\gamma}\left(\frac{\omega_{+}-\omega_{-}}{y-t}\right)^{\alpha}|\sigma(t, y)| d y,
\end{aligned}
$$

where we used the fact that $\mathcal{R} \in \mathcal{V}$.

Note that all the quantities inside the brackets are positive and that in $\partial_{\omega_{-}}^{2} \mathcal{R}_{0}\left(t, s ; \omega_{-}\right.$, $\left.\omega_{+}\right)$an example for the worst singular term is (see the Appendix)

$$
\frac{\left(\omega_{+}-s\right)^{2}\left(\omega_{+}-t\right)^{2}}{(s-t)^{\frac{5}{2}}\left(\omega_{+}-\omega_{-}\right)^{\frac{7}{2}}}\left(\ln \left(\frac{\left(s-\omega_{-}\right)\left(\omega_{+}-t\right)}{(s-t)\left(\omega_{+}-\omega_{-}\right)}\right)+\frac{(s-t)^{2}\left(\omega_{+}-\omega_{-}\right)^{2}}{\left(s-\omega_{-}\right)^{2}\left(\omega_{+}-t\right)^{2}}\right) .
$$

So finally we have to prove

$$
\begin{gathered}
\int_{s}^{\omega_{+}} \frac{\left(\omega_{+}-y\right)^{2}\left(\omega_{+}-t\right)^{2}}{(y-t)^{\frac{5}{2}}\left(\omega_{+}-\omega_{-}\right)^{\frac{7}{2}}}\left(\ln \left(\frac{\left(y-\omega_{-}\right)\left(\omega_{+}-t\right)}{(y-t)\left(\omega_{+}-\omega_{-}\right)}\right)\right. \\
\left.+\frac{(y-t)^{2}\left(\omega_{+}-\omega_{-}\right)^{2}}{\left(y-\omega_{-}\right)^{2}\left(\omega_{+}-t\right)^{2}}\right)\left(\frac{s-\omega_{-}}{s-t}\right)^{\gamma}\left(\frac{\omega_{+}-\omega_{-}}{y-t}\right)^{\alpha}|\sigma(t, y)| d y<+\infty .
\end{gathered}
$$

Thanks to Lemma 5.2, if $l>3+k$, we can assume $|\sigma(x, y)|=O\left(|y-x|^{k}\right)$ and $|\rho(x, y)|=O\left(|y-x|^{k}\right)$. 
Now, note that $\frac{y-\omega_{-}}{y-t} \leq 1$ and $\frac{\omega_{+}-y}{\omega_{+}-\omega_{-}} \leq 1$ implies, respectively,

$$
\ln \left(\frac{\left(y-\omega_{-}\right)\left(\omega_{+}-t\right)}{(y-t)\left(\omega_{+}-\omega_{-}\right)}\right) \leq \ln \left(\frac{\left(\omega_{+}-t\right)}{\left(\omega_{+}-\omega_{-}\right)}\right)
$$

and

We have to estimate

$$
\frac{\left(\omega_{+}-y\right)^{2}}{\left(\omega_{+}-\omega_{-}\right)^{\frac{7}{2}}} \leq \frac{1}{\left(\omega_{+}-\omega_{-}\right)^{\frac{3}{2}}} .
$$

$$
\begin{gathered}
\left(\omega_{+}-\omega_{-}\right)^{\alpha-\frac{3}{2}}\left(\omega_{+}-t\right)^{2}\left(\left|\ln \left(\omega_{+}-t\right)\right|+\left|\ln \left(\omega_{+}-\omega_{-}\right)\right|\right)\left(\frac{s-\omega_{-}}{s-t}\right)^{\gamma} \int_{s}^{\omega_{+}}(y-t)^{k-\left(\alpha+\frac{5}{2}\right)} d y \\
+\left(\omega_{+}-\omega_{-}\right)^{\alpha+\frac{1}{2}}\left(\frac{s-\omega_{-}}{s-t}\right)^{\gamma} \int_{s}^{\omega_{+}} \frac{(y-t)^{k-\left(\alpha+\frac{1}{2}\right)}}{\left(y-\omega_{-}\right)^{2}} d y=(a)+(b) .
\end{gathered}
$$

As $\frac{s-\omega_{-}}{s-t} \leq 1,(a)$ is finite if $\alpha>\frac{3}{2}, \gamma \geq 0$ and $k \geq \alpha+\frac{3}{2}$.

Now we analyse $(b)$. Integrating by parts, we have

$$
\begin{gathered}
\left(\omega_{+}-\omega_{-}\right)^{\alpha+\frac{1}{2}}\left(\frac{s-\omega_{-}}{s-t}\right)^{\gamma} \int_{s}^{\omega_{+}} \frac{(y-t)^{k-\left(\alpha+\frac{1}{2}\right)}}{\left(y-\omega_{-}\right)^{2}} d y \\
=\left(\omega_{+}-\omega_{-}\right)^{\alpha+\frac{1}{2}}\left(\frac{s-\omega_{-}}{s-t}\right)^{\gamma}\left(-\frac{\left(\omega_{+}-t\right)^{k-\left(\alpha+\frac{1}{2}\right)}}{\omega_{+}-\omega_{-}}+\frac{(s-t)^{k-\left(\alpha+\frac{1}{2}\right)}}{s-\omega_{-}}\right) \\
+\left(k-\left(\alpha+\frac{1}{2}\right)\right)\left(\omega_{+}-\omega_{-}\right)^{\alpha+\frac{1}{2}}\left(\frac{s-\omega_{-}}{s-t}\right)^{\gamma} \int_{s}^{\omega_{+}} \frac{(y-t)^{k-\left(\alpha+\frac{3}{2}\right)}}{\left(y-\omega_{-}\right)} d y .
\end{gathered}
$$

The worst case is the last term, so we concentrate on that one.

First we note that we can decompose

$$
\frac{(y-t)^{k-\left(\alpha+\frac{3}{2}\right)}}{\left(y-\omega_{-}\right)}=\frac{(y-t)^{\xi}(y-t)^{p}}{\left(y-\omega_{-}\right)}=(y-t)^{\xi}\left(P(y)+\frac{\left(\omega_{-}-t\right)^{p}}{\left(y-\omega_{-}\right)}\right),
$$

where $P(y)$ is a polynomial in $y, p=\left[k-\left(\alpha+\frac{3}{2}\right)\right] \geq 1$ and $\xi=k-\left(\alpha+\frac{3}{2}\right)-p$. (Here $[x]$ is the floor of $x$.)

So we have

$$
\begin{gathered}
\left(\omega_{+}-\omega_{-}\right)^{\alpha+\frac{1}{2}}\left(\frac{s-\omega_{-}}{s-t}\right)^{\gamma} \int_{s}^{\omega_{+}} \frac{(y-t)^{k-\left(\alpha+\frac{3}{2}\right)}}{\left(y-\omega_{-}\right)} d y \\
\leq\left(\omega_{+}-\omega_{-}\right)^{\alpha+\frac{1}{2}}\left(\frac{s-\omega_{-}}{s-t}\right)^{\gamma}\left(\omega_{+}-t\right)^{\xi} \int_{s}^{\omega_{+}} P(y) d y \\
+\left(\omega_{+}-\omega_{-}\right)^{\alpha+\frac{1}{2}}\left(\frac{s-\omega_{-}}{s-t}\right)^{\gamma}\left(\omega_{+}-t\right)^{\xi}\left(\omega_{-}-t\right)^{p}\left|\ln \left(\omega_{+}-\omega_{-}\right)\right| \\
+\left(\omega_{+}-\omega_{-}\right)^{\alpha+\frac{1}{2}}\left(\frac{s-\omega_{-}}{s-t}\right)^{\gamma}\left(\omega_{+}-t\right)^{\xi}\left(\omega_{-}-t\right)^{p}\left|\ln \left(s-\omega_{-}\right)\right| .
\end{gathered}
$$

Again the worst condition comes from the last term. Given $\delta \in(0,1) \subset \mathbb{R}$, we can write such a term as

$$
\left(\omega_{+}-\omega_{-}\right)^{\alpha+\frac{1}{2}}\left(\frac{s-\omega_{-}}{s-t}\right)^{\gamma-\delta}\left(\omega_{+}-t\right)^{\xi} \frac{\left(\omega_{-}-t\right)^{p}}{(s-t)^{\delta}}\left(s-\omega_{-}\right)^{\delta}\left|\ln \left(s-\omega_{-}\right)\right|
$$

that is finite as $p \geq \delta$. So we need to require $k-\left(\alpha+\frac{3}{2}\right) \geq 1$, that is, $k \geq \alpha+\frac{5}{2}$. 
If we take into account the previous condition $\alpha>\frac{3}{2}$, we finally have $k>4$, and so $l>7$.

5.2. $C^{2}$ East type entropies. Our aim is to find some conditions on the Goursat data in order to obtain solutions to (5.1) whose derivatives, up to the second order, are bounded in

$$
J^{*}:=\left\{\left(\omega_{-}, \omega_{+}\right) \mid \omega_{-}^{*} \leq \omega_{-} \leq 0 \leq \omega_{+} \leq \omega_{+}^{*}\right\} .
$$

Now we can state the main result of this section:

TheOREM 5.6. Assume hypothesis (1.3) with $l>7$ and consider the Goursat problem (5.1). If $-\delta<\omega_{-}^{*} \leq \omega_{+}^{*}<\delta$, for a small positive constant $\delta$, there exists a family of Goursat data

$$
\begin{gathered}
\Theta:=\left\{\theta_{-} \in C^{2}(\mathbb{R}) \mid \int_{\omega_{-}^{*}}^{-\delta}\left((-t)^{-\frac{3}{2}}\left(\theta_{-}^{\prime}(t)-\frac{1}{2 t} \theta_{-}(t)\right)\right) d t=0\right. \text { and } \\
\left.\int_{\omega_{-}^{*}}^{-\delta}\left((-t)^{-\frac{5}{2}}\left(\theta_{-}^{\prime}(t)-\frac{1}{2 t} \theta_{-}(t)\right)\right) d t=0\right\}
\end{gathered}
$$

such that, for every $\theta_{-} \in \Theta$, problem (5.1) has a unique solution $\eta\left(\omega_{-}, \omega_{+}\right) \in C^{2}\left(J^{*} \backslash\right.$ $(0,0))$.

Moreover $\eta$ and its derivatives in $\omega_{ \pm}$, up to second order, are bounded on bounded sets in $J^{*}$.

Proof. Recall the integral representation (5.2) for East type entropies:

$$
\eta\left(\omega_{-}, \omega_{+}\right)=\int_{\omega_{-}^{*}}^{\omega_{-}} \mathcal{R}\left(t, 0 ; \omega_{-}, \omega_{+}\right)\left(\theta_{-}^{\prime}(t)-\frac{\partial_{\omega_{-}} \lambda_{+}(t, 0)}{\lambda_{+}(t, 0)-\lambda_{-}(t, 0)} \theta_{-}(t)\right) d t
$$

that, for the unperturbed case (see the Appendix for details), becomes

$$
\eta_{0}\left(\omega_{-}, \omega_{+}\right)=\int_{\omega_{-}^{*}}^{\omega_{-}}\left(\frac{\omega_{+}-\omega_{-}}{-t}\right)^{\frac{1}{2}} H\left(\frac{\omega_{+}\left(\omega_{-}-t\right)}{-t\left(\omega_{+}-\omega_{-}\right)}\right)\left(\theta_{-}^{\prime}(t)-\frac{1}{2 t} \theta_{-}(t)\right) d t .
$$

In Lemma 5.2 and Theorem 5.5. respectively, we proved

$$
\lim _{\left|\omega_{+}-\omega_{-}\right| \longrightarrow 0} \rho\left(\omega_{-}, \omega_{+}\right)=\lim _{\left|\omega_{+}-\omega_{-}\right| \longrightarrow 0} \frac{1}{2\left(\left(\omega_{+}\right)_{0}-\left(\omega_{-}\right)\right)_{0}}-\frac{\partial_{\omega_{-}} \lambda_{+}}{\lambda_{+}-\lambda_{-}}=0
$$

and

$$
\lim _{\left|\omega_{+}-\omega_{-}\right| \longrightarrow 0}\left\|\mathcal{R}-\mathcal{R}_{0}\right\|_{\mathcal{V}}=0 .
$$

Thanks to these results, we can prove that there exists a constant $c$ such that, in a small neighbourhood of the origin,

$$
\left|\eta\left(\omega_{-}, \omega_{+}\right)-\eta(0,0)\right| \leq c\left|\eta_{0}\left(\omega_{-}, \omega_{+}\right)-\eta_{0}(0,0)\right| .
$$

Now, by Proposition A.1 (see the Appendix) we can conclude $\eta\left(\omega_{-}, \omega_{+}\right) \in C\left(J^{*}\right)$.

Using the analogous estimates for the derivatives of first and second order proven in Theorem 5.5 and the results in Proposition A.1, we can close the proof. 
For our purposes we will need the regularity of entropies in the state variables. The result is non-trivial because the transformation $T$ from the state variables to the Riemann invariant is not regular in $(0,0)$.

So we need to prove

THEOREM 5.7. Assume hypothesis (1.3).

Let $\eta\left(\omega_{-}, \omega_{+}\right)$be a solution for the Goursat problem (5.1) with $\theta \in \Theta$.

If $-\delta<\omega_{-} \leq \omega_{+}<\delta$, for a small positive constant $\delta$, then $\eta(u, v):=T^{-1}\left(\eta\left(\omega_{-}, \omega_{+}\right)\right)$ $\in C^{2}\left(\mathbb{R}^{2} \backslash(0,0)\right)$

Moreover $\eta$ and its derivatives in $u$, $v$, up to second order, are bounded on bounded sets in the state space $I$.

Proof. It will be enough to observe that, under hypothesis (1.3),

$$
\begin{aligned}
\frac{\partial \omega_{ \pm}}{\partial u} & =\left(\frac{\partial \omega_{ \pm}}{\partial u}\right)_{0}+O\left((|u|+|v|)^{l-1}\right), \\
\frac{\partial \omega_{ \pm}}{\partial v} & =\left(\frac{\partial \omega_{ \pm}}{\partial v}\right)_{0}+O\left((|u|+|v|)^{l-1}\right),
\end{aligned}
$$

where $\left(\partial \omega_{ \pm} / \partial u\right)_{0}$ and $\left(\partial \omega_{ \pm} / \partial v\right)_{0}$ are the derivatives of the Riemann invariants associated to the unperturbed system.

Now using the chain rule and Theorem 5.6 we have the result.

Finally it will also be useful to establish the following representation for an entropy -entropy flux pair $(\eta, q)$.

Corollary 5.8. Suppose $(\eta, q)$ is an entropy-entropy flux pair, where $\eta$ is an entropy of type East or West with limit $\omega_{-} *$. Then $\eta$ and $q$ have the following representations:

$$
\begin{aligned}
& \eta\left(\omega_{-}, \omega_{+}\right)=A\left(\omega_{-}, \omega_{+}\right) \theta_{-}\left(\omega_{-}\right)+\int_{\omega_{-}^{*}}^{\omega_{-}} B\left(x, \omega_{-}, \omega_{+}\right) \theta_{-}(x) d x, \\
& q\left(\omega_{-}, \omega_{+}\right)=C\left(\omega_{-}, \omega_{+}\right) \theta_{-}\left(\omega_{-}\right)+\int_{\omega_{-}^{*}}^{\omega_{-}} D\left(x, \omega_{-}, \omega_{+}\right) \theta_{-}(x) d x,
\end{aligned}
$$

where $A, B, C$ and $D$ are smooth functions in the variables $x, \omega_{-}, \omega_{+}$whenever $x<0$ and $\omega_{-}<0$.

Proof. Again using the integral representation (5.2) for $\eta$, the proof follows from Lemma 5.2. Theorem 5.5 and the results in the Appendix.

6. Strong convergence by compensated compactness. In this section we will show that the sequence of solutions $\left\{\left(u^{\varepsilon}, v^{\varepsilon}\right)\right\}_{\varepsilon>0}$ of system (3.1) that we obtained in Section 3 converges strongly to a solution $(u, v)$ of (1.1) when $\varepsilon \rightarrow 0$.

We first introduce the Young measure describing the weak convergence of the sequence $\left\{\left(u^{\varepsilon}, v^{\varepsilon}\right)\right\}_{\varepsilon>0}$. Then, using the results in Section 5, we set up a commutation relation and a new representation for the entropy-entropy flux pair. Finally we reduce the Young measure to a point mass so that we can apply Tartar's result ([31]) and obtain strong convergence. 
Before starting to analyse our case, we recall the most important results of the basic theory of compensated compactness. We will refer mainly to [26], chap. 9 .

Let $\Omega$ be an open set of $\mathbb{R}^{n}$ and $K$ a closed set of $\mathbb{R}^{p}$.

We consider a sequence of functions $u_{k}: \Omega \longrightarrow \mathbb{R}^{p}$ such that $u_{k}(x) \in K$ for almost all $x \in \Omega$.

Proposition 6.1 (Proposition 9.1.7, p. 47 of [26]). Let $\left\{U_{k}\right\}_{k \in \mathbb{N}}$ be a sequence in $L^{\infty}(\Omega)$, convergent in the sense of Young to the measure $\nu$. Let $U$ be its weak-star limit. The following assertions are equivalent:

(1) There exists $p \in[1, \infty]$ such that $\left\|U_{k}-U\right\|_{L^{p}(\omega)} \rightarrow 0$ for every bounded set $\omega \in \Omega$.

(2) The same statement is true for all $p \in[1, \infty)$.

(3) For almost all $x \in \Omega, \nu_{x}$ is a Dirac mass.

Next we recall Tartar's result:

Proposition 6.2 (Proposition 9.2.2, p. 57 of [26]). We consider a physical system (1.2) and we suppose that a sequence $\left(U^{\varepsilon}\right)_{n \in \mathbb{N}}$ of solutions of its viscous approximation for $\varepsilon=\varepsilon_{n} \rightarrow 0^{+}$has values in a compact set and converges in the sense of Young to $\nu$.

Then for all entropy-entropy flux pairs $\left(\eta_{j}, q_{j}\right), j=1,2$, of class $C^{2}$, we have

$$
\left\langle\nu_{x, t}, \eta_{1} q_{2}-\eta_{2} q_{1}\right\rangle=\left\langle\nu_{x, t}, \eta_{1}\right\rangle\left\langle\nu_{x, t}, q_{2}\right\rangle-\left\langle\nu_{x, t}, \eta_{2}\right\rangle\left\langle\nu_{x, t}, q_{1}\right\rangle,
$$

for almost all $(x, t) \in \mathbb{R} \times \mathbb{R}^{+}$.

Let $\left(U^{\varepsilon}\right)_{\varepsilon>0}$ be a sequence of solutions for the viscous approximation to (1.2).

From the results in Section 3 we know that, for every $\varepsilon>0$ such a solution exists, global in time, and the sequence $\left(U^{\varepsilon}\right)_{\varepsilon>0}$ is uniformly bounded with respect to $\varepsilon$.

So we can apply the result of Theorem 9.1.5, p. 44 of [26] and describe the weak convergence of $U^{\varepsilon}$ through a Young measure $\nu=\nu_{(x, t)}$ :

$$
g\left(U^{\varepsilon}(x, t)\right) \rightarrow \int g(s) d \nu_{(x, t)}(s)=:\left\langle\nu_{(x, t)}, g\right\rangle,
$$

for any continuous function $g$.

Our goal is to reduce this Young measure to a Dirac mass, then apply Proposition 6.1 and finally obtain strong convergence for $\left(U^{\varepsilon}\right)_{\varepsilon>0}$.

In order to reduce the Young measure we will need the commutation relation in Proposition 6.2 .

To this end, using the entropy $\eta^{*}$ from Remark 4.2, we can prove in the standard way the following:

Lemma 6.3. Let $U^{\varepsilon}=\left(u^{\varepsilon}, v^{\varepsilon}\right)$ be a $L^{2}$ solution for the viscous approximation (3.1). Then there exists a constant $M>0$ such that, for every $\varepsilon>0$,

$$
2 \varepsilon \int_{0}^{T} \int_{-\infty}^{+\infty}\left(\left(u^{\varepsilon}\right)_{x}^{2}+\left(v^{\varepsilon}\right)_{x}^{2}\right) d x d t \leq M
$$

With this result we can prove: 
Proposition 6.4. Let $U^{\varepsilon}=\left(u^{\varepsilon}, v^{\varepsilon}\right)$ be a $L^{2}$ solution to (3.1), and $(\eta, q)$ a $C^{2}$ entropyentropy flux pair for (1.2). Then $\left(\eta\left(U^{\varepsilon}\right)_{t}+q\left(U^{\varepsilon}\right)_{x}\right)_{\varepsilon>0}$ is relatively compact in $H_{\text {loc }}^{-1}(\mathbb{R} \times$ $\left.\mathbb{R}^{+}\right)$.

Proof. It follows from the result of Murat (Lemma 9.2.1, p. 57 of [26]), using Theorem 5.7 and Lemma 6.3

Now the hypotheses of Tartar (Proposition 6.2) are satisfied by all the couples of entropy-entropy flux pairs $\left(\eta_{j}, q_{j}\right), j=1,2$, of class $C^{2}$ so that we have the commutation relation

$$
\left\langle\nu_{x, t}, \eta_{1} q_{2}-\eta_{2} q_{1}\right\rangle=\left\langle\nu_{x, t}, \eta_{1}\right\rangle\left\langle\nu_{x, t}, q_{2}\right\rangle-\left\langle\nu_{x, t}, \eta_{2}\right\rangle\left\langle\nu_{x, t}, q_{1}\right\rangle,
$$

for almost all $(x, t) \in \mathbb{R} \times \mathbb{R}^{+}$.

To reduce the Young measure to a Dirac mass is now equivalent to proving that the only non-zero solution to (6.2) is a Dirac mass.

6.1. The reduction of the Young measure. First we notice that, by the geometrical construction in Section 2, if we conclude that the Young measure $\nu$ is a point mass in the $\left(\omega_{-}, \omega_{+}\right)$plane, then $\nu$ is at most the sum of two $\delta$-functions in the $(u, v)$ plane. By using the hypothesis $\left(u_{0}, v_{0}\right) \in I^{+}$and the result from Corollary 3.5 , we have that $\nu$ is a point mass in the $(u, v)$ plane too.

Therefore it is sufficient to prove our result in the Riemann invariant coordinates. Now let

$$
R=\left[\omega_{-}^{-}, 0\right] \times\left[0, \omega_{+}^{+}\right]
$$

be the minimal rectangle in the $\left(\omega_{-}, \omega_{+}\right)$plane containing the support of $\nu$. We will just analyse the most complex case, that is, when $R$ contains the umbilic point and $\omega_{-}^{-}<0<\omega_{+}^{+}$(so that $R$ is not just a line parallel to one of the axes).

After some preliminary remarks, we divide our proof in two parts: first we show that the support of $\nu$ concentrates on the four corners of $R$, and then we reduce again to get just one Dirac mass.

Let $\omega_{-}^{-}<-\delta<0$, where $\delta>0$ is the positive constant that gives us the zero moment condition in Theorem 5.6 .

Let $\gamma \in \mathbb{R}$ satisfy $\omega_{-}^{-}<-\gamma<-\delta$, and $\varepsilon^{\prime \prime}=\varepsilon^{\prime \prime}(\gamma, \delta)$ such that

$$
\omega_{-}^{-}<\omega_{-}^{*}<\gamma<\omega_{-}^{\sharp}<-\delta,
$$

where $\omega_{-}^{*}=\gamma-\varepsilon^{\prime \prime}$ and $\omega_{-}^{\sharp}=\gamma+\varepsilon^{\prime \prime}$.

Now we can use the results in [13] and generalise to our case the theory developed by [25] for strictly hyperbolic systems, and prove the following:

Lemma 6.5. Let $(\eta, q)$ be an entropy-entropy flux pair of type East with limit $\omega_{-}^{*}$ and $(\bar{\eta}, \bar{q})$ be an entropy-entropy flux pair of type West with limit $\omega_{-}^{\sharp}$.

Let $\theta_{-}$and $\bar{\theta}_{-}$be their Goursat data (as in the hypotheses of Theorem [5.6).

Let $\theta_{-}(\gamma), \bar{\theta}_{-}(\gamma), \theta_{-}^{\prime}(\gamma) \bar{\theta}_{-}^{\prime}(\gamma), \theta_{-}^{\prime \prime}(\gamma)$ and $\bar{\theta}_{-}^{\prime \prime}(\gamma)$ be fixed positive constants independent of $\varepsilon^{\prime \prime}$.

(i) Suppose that for $\varepsilon^{\prime \prime}>0$ small enough, if $\langle\nu, \eta \bar{q}-\bar{\eta} q\rangle=0$, then

$$
\operatorname{supp}(\nu) \cap\left\{\left(\omega_{-}, \omega_{+}\right) \mid \omega_{+} \geq 0, \omega_{-}=\gamma\right\}=\emptyset .
$$


(ii) Let $\omega_{-}^{-} \leq \omega_{-}^{*} \leq-\delta$. Then there exists a constant $c$, independent from $\omega_{-}^{*}$, such that

$$
\langle\nu, q\rangle=c\langle\nu, \eta\rangle .
$$

(iii) Fix $\delta>0$. Let $\omega_{-}^{\sharp}$ satisfy $\omega_{-}^{-}<\omega_{-}^{\sharp}<-\delta<0$. If, for every East type entropy $\bar{\eta}$ with limit $\omega_{-}^{\sharp}$ we have

$$
\langle\nu, \bar{\eta}\rangle=0
$$

then

$$
\operatorname{supp}(\nu) \cap\left\{\left(\omega_{-}, \omega_{+}\right) \mid \omega_{+} \geq 0, \omega_{-}^{\sharp} \leq \omega_{-} \leq-\delta\right\}=\emptyset .
$$

Thanks to these results it is possible to prove:

TheOREM 6.6. The Young measure $\nu$ as defined in (6.1) is a point mass in the plane $\left(\omega_{-}, \omega_{+}\right)$.

Proof. Following D. Serre ([25]), we divide our proof in three steps.

STEP 1. Reduction to four $\delta$-functions. Let $\delta>0$ be small enough so that $\omega_{-}^{-}<-\delta$, and define

$$
\omega_{-}(\delta):=\inf \left\{y \mid \omega_{-}^{-} \leq y \leq-\delta, \operatorname{supp}(\nu) \cap\left\{\left(\omega_{-}, \omega_{+}\right) \mid y<\omega_{-}<-\delta\right\}=\emptyset\right\} .
$$

Under our assumptions, it is easy to prove that

$$
\operatorname{supp}(\nu) \subset\left\{\left(\omega_{-}, \omega_{+}\right) \mid 0 \leq \omega_{+} \leq \omega_{+}^{+} \text {and } \omega_{-}=\omega_{-}^{-} \text {or }-\delta \leq \omega_{-} \leq 0\right\}
$$

and

$$
\operatorname{supp}(\nu) \subset\left\{\left(\omega_{-}, \omega_{+}\right) \mid \omega_{-}^{-} \leq \omega_{-} \leq 0 \text { and } \omega_{+}=\omega_{+}^{+} \text {or } 0 \leq \omega_{+} \leq \delta\right\} .
$$

The support of $\nu$ is so concentrated on the square $\left\{\left(\omega_{-}, \omega_{+}\right) \mid-\delta \leq \omega_{-} \leq 0,0 \leq \omega_{+} \leq\right.$ $\delta\}$ and on the points $\left(\omega_{-}^{-}, 0\right),\left(0, \omega_{+}^{+}\right),(0,0)$ and $\left(\omega_{-}^{-}, \omega_{+}^{+}\right)$.

If we let $\delta \rightarrow 0^{+}$we obtain that the support of $\nu$ is concentrated on the four corners of $R$.

STEP 2. Reduction from 4 to $3 \delta$-functions. Let $A_{i}, i=1,2,3,4$, be the four corners of $R$, with $A_{1}=\left(\omega_{-}^{-}, 0\right), A_{2}=\left(\omega_{-}^{-}, \omega_{+}^{+}\right), A_{3}=(0,0)$ and $A_{4}=\left(0, \omega_{+}^{+}\right)$. We know that

$$
\nu=\sum_{1 \leq i \leq 4} \beta_{i} \delta_{A_{i}}
$$

where $\beta_{i} \geq 0$ for every $i$, and $\sum_{1 \leq i \leq 4} \beta_{i}=1$. Assume $\beta_{i} \neq 0$ for every $i$.

If we consider two entropy-entropy flux pairs $\left(\eta_{1}, q_{1}\right)$ and $\left(\eta_{2}, q_{2}\right)$, applying the commutation relation (6.2) we get

$$
\sum_{i, j=1}^{4} \beta_{i} \beta_{j}\left\{\left(\eta_{1}\left(A_{i}\right)-\eta_{1}\left(A_{j}\right)\right)\left(q_{2}\left(A_{i}\right)-q_{2}\left(A_{j}\right)\right)-\left(\eta_{2}\left(A_{i}\right)-\eta_{2}\left(A_{j}\right)\right)\left(q_{1}\left(A_{i}\right)-q_{1}\left(A_{j}\right)\right)\right\}=0 .
$$

Now let $\Theta$ be an application with values in $\mathbb{R}^{8}$ defined on the pairs $(\eta, q)$ in the following way:

$$
\Theta(\eta, q)=\left(\eta\left(A_{1}\right), \ldots, \eta\left(A_{4}\right), q\left(A_{1}\right), \ldots, q\left(A_{4}\right)\right)
$$


Its image is $X \subset \mathbb{R}^{8}$. Also define a linear form $f$ on $\mathbb{R}^{8} \times \mathbb{R}^{8}$ :

$$
f\left(\left(x_{1}, y_{1}\right),\left(x_{2}, y_{2}\right)\right)=\sum_{i, j=1}^{4} \beta_{i} \beta_{j}\left\{\left(x_{1 i}-x_{1 j}\right)\left(y_{2 i}-y_{2 j}\right)-\left(x_{2 i}-x_{2 j}\right)\left(y_{1 i}-y_{1 j}\right)\right\} .
$$

If $\beta_{i} \neq 0$ for every $i$, then $f$ is an antisymmetric form of rank 6 , of the same form as (6.3), so that we have

$$
(a, b) \in X \times X \Rightarrow f(a, b)=0,
$$

that also implies $\operatorname{dim} X \leq 5$.

Now let $1 \leq l \leq 4$. As the quadratic forms $(\eta, q) \rightarrow \eta\left(A_{i}\right)$ and $(\eta, q) \rightarrow q\left(A_{i}\right), i \neq l$, are six linearly dependent forms on the set of pairs $(\eta, q)$, there exist real numbers $a_{i l}$, $b_{i l}, i \neq l$, not all equal to zero, such that

$$
\sum_{i \neq l} a_{i l} q\left(A_{i}\right)+b_{i l} \eta\left(A_{i}\right)=0
$$

for every pair $(\eta, q)$.

We now consider an entropy $\eta$ of type East, that verifies in particular $\theta_{-}=0$ if $\omega_{-}=\omega_{-}^{*}$ (see Definition 4.3), and the associated flux function $q$. Then we have

$$
\eta\left(A_{1}\right)=\eta\left(A_{2}\right)=q\left(A_{1}\right)=q\left(A_{2}\right)=0,
$$

and from (6.4), for $l=4$, we have $a_{34} q\left(A_{3}\right)+b_{34} \eta\left(A_{3}\right)=0$.

From this follows that

$$
\begin{aligned}
\left(b_{34}+a_{34} \lambda_{-}\left(A_{3}\right)\right) \eta\left(A_{3}\right) & =-a_{34} \lambda_{-}\left(A_{3}\right) \eta\left(A_{3}\right)-a_{34} \int_{A_{1}}^{A_{3}} \frac{\partial q}{\partial \omega_{-}} d \omega_{-} \\
& =a_{34} \int_{A_{1}}^{A_{3}} \eta \frac{\partial \lambda_{-}}{\partial \omega_{-}} d \omega_{-} .
\end{aligned}
$$

Notice that once we have $\eta\left(A_{1}\right)=0$, the restriction of $\eta$ to $A_{1} A_{3}$ is arbitrary, so we have

$$
\begin{aligned}
a_{34} \frac{\partial \lambda_{-}}{\partial \omega_{-}} & =0 \quad \text { on } \quad A_{1} A_{3}, \\
b_{34}+a_{34} \lambda_{-}\left(A_{3}\right) & =0 .
\end{aligned}
$$

Using an entropy of type North we get

$$
\begin{aligned}
a_{24} \frac{\partial \lambda_{+}}{\partial \omega_{+}} & =0 \quad \text { on } \quad A_{1} A_{2}, \\
b_{24}+a_{24} \lambda_{+}\left(A_{3}\right) & =0 .
\end{aligned}
$$

If $\frac{\partial \lambda_{-}}{\partial \omega_{-}} \neq 0$ on $A_{1} A_{3}$ and $\frac{\partial \lambda_{+}}{\partial \omega_{+}} \neq 0$ on $A_{1} A_{2}$, then from (6.6) and (6.7) we have

$$
a_{34}=a_{24}=b_{34}=b_{24}=0,
$$

and so, from (6.4) with $l=4$, we have $a_{14} \eta\left(A_{1}\right)+b_{14}\left(A_{1}\right) q\left(A_{1}\right)=0$ for all pairs $(\eta, q)$. This is absurd.

Since from the genuine non-linearity of (1.1) in the interior of $R$ and from Lemma 2.6 we have that $\partial \lambda_{-} / \partial \omega_{-}$and $\partial \lambda_{+} / \partial \omega_{+}$are non-zero everywhere, the only possible explanation is that there exists an $i, 1 \leq i \leq 4$, such that $\beta_{i}=0$. 
STEP 3. Reduction to one $\delta$-function. In what follows, for every function $f$ we will use the notation $f\left(A_{i}\right)=f_{i}$.

We now know that

$$
\nu=\beta_{1} A_{1}+\beta_{2} A_{2}+\beta_{3} A_{3}+\beta_{4} A_{4}
$$

where, at least for one index $i, \beta_{i}=0$.

Assume $\beta_{4}=0$. Then

$$
\nu=\beta_{1} A_{1}+\beta_{2} A_{2}+\beta_{3} A_{3} .
$$

Let $(\eta, q)$ be a West type entropy-entropy flux pair, with limit $\frac{1}{2} \omega_{-}^{-}$, and $\left(\eta^{k}, q^{k}\right)$ an entropy-entropy flux pair as constructed in Remark 4.2 .

We choose the Goursat data so that $\eta_{1}=\eta_{2}=q_{1}=q_{2}=0$, and combining (6.8) with the commutation relation we get

$$
\left(\beta_{3}-\beta_{3}^{2}\right)\left(\eta_{3} q_{3}^{k}-\eta_{3}^{k} q_{3}\right)=\beta_{3} \eta_{3}\left(\beta_{1} q_{1}^{k}+\beta_{2} q_{2}^{k}\right)-\beta_{3} q_{3}\left(\beta_{1} \eta_{1}^{k}+\beta_{2} \eta_{2}^{k}\right) .
$$

We can assume $\beta_{1}+\beta_{2}>0$ and choose $\left(\eta^{k}, q^{k}\right)$ such that

$$
\beta_{1} q_{1}^{k}+\beta_{2} q_{2}^{k}=0=\beta_{1} \eta_{1}^{k}+\beta_{2} \eta_{2}^{k} .
$$

So we have $\left(\beta_{3}-\beta_{3}^{2}\right)\left(\eta_{3} q_{3}^{k}-\eta_{3}^{k} q_{3}\right)=0$.

As $(\eta, q)$ is an arbitrary West type entropy-entropy flux pair and $\eta^{k}, q^{k}$ is an arbitrary entropy-entropy flux pair from Remark 4.2, this implies

$$
\beta_{3}-\beta_{3}^{2}=0 \text {. }
$$

This means

$$
\beta_{4}=0 \Rightarrow \beta_{3}=0 \text { or } \beta_{3}=1 .
$$

In a similar way we can find

$$
\begin{aligned}
& \beta_{1}=0 \Rightarrow \beta_{4}=0 \text { or } \beta_{4}=1, \\
& \beta_{3}=0 \Rightarrow \beta_{4}=0 \text { or } \beta_{4}=1
\end{aligned}
$$

and

$$
\beta_{2}=0 \Rightarrow \beta_{4}=0 \text { or } \beta_{4}=1 \text {. }
$$

At this point we know that

$$
\nu=\rho_{1} \delta_{P}+\rho_{2} \delta_{Q},
$$

where $P$ and $Q$ are two of the four corners of $R$ and $\rho_{1}, \rho_{2}$ are non-negative constants such that $\rho_{1}+\rho_{2}=1$. We have finally to prove that $\nu$ can be reduced to one $\delta$-function.

Let $\left(\eta^{k}, q^{k}\right)$ and $\left(\eta^{j}, q^{j}\right)$ be two entropy-entropy flux pairs from Remark 4.2 and assume $P \neq(0,0)$. Choosing the appropriate constants, we can have

$$
\eta^{k}(Q)=q^{k}(Q)=0=\eta^{j}(Q)=q^{j}(Q) .
$$

The commutation relation gives $\left(\rho_{1}-\rho_{1}^{2}\right)\left(\eta^{k} q^{j}-\eta^{j} q^{k}\right)=0$.

As $\left(\eta^{k}, q^{k}\right)$ and $\left(\eta^{j}, q^{j}\right)$ are arbitrary and $P \neq(0,0)$, this implies $\rho_{1}-\rho_{1}^{2}=0$, that is, $\rho_{1}=0$ or $\rho_{1}=1$. 
Appendix A. The unperturbed case. We now recall some results from Kan ([13], [14]).

In the unperturbed case the entropy equation (4.2) becomes

$$
\frac{\partial^{2} \eta_{0}}{\partial \omega_{+} \partial \omega_{-}}+\frac{1}{2\left(\left(\omega_{+}\right)_{0}-\left(\omega_{-}\right)_{0}\right)}\left(\frac{\partial \eta_{0}}{\partial \omega_{+}}-\frac{\partial \eta_{0}}{\partial \omega_{-}}\right)=0
$$

where $\left(\omega_{-}\right)_{0}$ and $\left(\omega_{+}\right)_{0}$ are the Riemann invariants associated to the unperturbed system:

$$
\begin{aligned}
& \left(\omega_{-}\right)_{0}=u-\sqrt{u^{2}+v^{2}}, \\
& \left(\omega_{+}\right)_{0}=u+\sqrt{u^{2}+v^{2}} .
\end{aligned}
$$

If $\mathcal{R}_{0}$ is the Riemann function associated to (A.1) it can be represented as

$$
\mathcal{R}_{0}\left(t, s ; \omega_{-}, \omega_{+}\right)=\left(\frac{\omega_{+}-\omega_{-}}{s-t}\right)^{\frac{1}{2}} H(w),
$$

where $w=\frac{\left(\omega_{+}-s\right)\left(\omega_{-}-t\right)}{(s-t)\left(\omega_{+}-\omega_{-}\right)}$and $H(w)$ is the hypergeometric function.

In what follows we summarize some properties about the singularities of $\mathcal{R}_{0}$ that were used in the proof of Theorem 5.5.

A.1. Singularities of the Riemann function. The hypergeometric function $H(w)$ is such that $H(0)=1$, it is analytic when $0 \leq w<1$ and in a neighbourhood of $w=1$ it can be represented in the form

$$
H(w)=A H_{1}(w-1)+B H_{1}(w-1) \ln (1-w)+H_{2}(w-1),
$$

where $H_{i}(\cdot)$ is analytical near 0 and $H_{1}(0)=1$.

Also note that

$$
H^{\prime}(w)=A H_{1}^{\prime}(w-1)+B H_{1}^{\prime}(w-1) \ln (1-w)-\frac{B H_{1}(w-1)}{1-w}+H_{2}^{\prime}(w-1)
$$

and

$$
H^{\prime \prime}(w)=A H_{1}^{\prime \prime}(w-1)+B H_{1}^{\prime \prime}(w-1) \ln (1-w)-2 \frac{B H_{1}^{\prime}(w-1)}{1-w}-\frac{B H_{1}(w-1)}{(1-w)^{2}}+H_{2}^{\prime \prime}(w-1) .
$$

In the proof of Theorem 5.5, we have to prove that

$$
\sup _{\mathcal{Q}_{\varepsilon}^{2}}\left|\partial_{\omega_{-}}^{i} \partial_{\omega_{+}}^{j} F\left(t, s ; \omega_{-}, \omega_{+}\right)\right|\left|s-\omega_{-}\right|^{\gamma}|s-t|^{\beta}\left|\omega_{+}-\omega_{-}\right|^{\alpha}
$$

is bounded. Problems arise near the origin, i.e. when $w=1$.

For instance, for the zero-order derivative term we have to consider the singularities of $\ln (1-w)$ in $H(w)$. So, for our purposes, instead of considering the whole expression for $\mathcal{R}_{0}$ we estimate only the term containing

$$
\left(\frac{\omega_{+}-\omega_{-}}{s-t}\right)^{\frac{1}{2}} \ln \left(\frac{\left(s-\omega_{-}\right)\left(\omega_{+}-t\right)}{(s-t)\left(\omega_{+}-\omega_{-}\right)}\right) .
$$

When deriving $\mathcal{R}_{0}$ up to the second order with respect to $\omega_{-}$and $\omega_{+}$, we obtain

$$
\begin{aligned}
& \frac{\left(\omega_{+}-s\right)\left(\omega_{+}-t\right)}{(s-t)^{\frac{3}{2}}\left(\omega_{+}-\omega_{-}\right)^{\frac{3}{2}}}\left(\ln \left(\frac{\left(s-\omega_{-}\right)\left(\omega_{+}-t\right)}{(s-t)\left(\omega_{+}-\omega_{-}\right)}\right)+\frac{(s-t)\left(\omega_{+}-\omega_{-}\right)}{\left(s-\omega_{-}\right)\left(\omega_{+}-t\right)}\right), \\
& \frac{\left(s-\omega_{-}\right)\left(\omega_{-}-t\right)}{(s-t)^{\frac{3}{2}}\left(\omega_{+}-\omega_{-}\right)^{\frac{3}{2}}}\left(\ln \left(\frac{\left(s-\omega_{-}\right)\left(\omega_{+}-t\right)}{(s-t)\left(\omega_{+}-\omega_{-}\right)}\right)+\frac{(s-t)\left(\omega_{+}-\omega_{-}\right)}{\left(s-\omega_{-}\right)\left(\omega_{+}-t\right)}\right),
\end{aligned}
$$




$$
\begin{gathered}
\frac{\left(\omega_{+}-s\right)^{2}\left(\omega_{+}-t\right)^{2}}{(s-t)^{\frac{5}{2}}\left(\omega_{+}-\omega_{-}\right)^{\frac{7}{2}}}\left(\ln \left(\frac{\left(s-\omega_{-}\right)\left(\omega_{+}-t\right)}{(s-t)\left(\omega_{+}-\omega_{-}\right)}\right)+\frac{(s-t)^{2}\left(\omega_{+}-\omega_{-}\right)^{2}}{\left(s-\omega_{-}\right)^{2}\left(\omega_{+}-t\right)^{2}}\right), \\
\frac{\left(s-\omega_{-}\right)^{2}\left(\omega_{-}-t\right)^{2}}{(s-t)^{\frac{5}{2}}\left(\omega_{+}-\omega_{-}\right)^{\frac{7}{2}}}\left(\ln \left(\frac{\left(s-\omega_{-}\right)\left(\omega_{+}-t\right)}{(s-t)\left(\omega_{+}-\omega_{-}\right)}\right)+\frac{(s-t)^{2}\left(\omega_{+}-\omega_{-}\right)^{2}}{\left(s-\omega_{-}\right)^{2}\left(\omega_{+}-t\right)^{2}}\right), \\
\frac{\left(\omega_{+}-s\right)\left(\omega_{+}-t\right)\left(s-\omega_{-}\right)\left(\omega_{-}-t\right)}{(s-t)^{\frac{5}{2}}\left(\omega_{+}-\omega_{-}\right)^{\frac{7}{2}}}\left(\ln \left(\frac{\left(s-\omega_{-}\right)\left(\omega_{+}-t\right)}{(s-t)\left(\omega_{+}-\omega_{-}\right)}\right)+\frac{(s-t)^{2}\left(\omega_{+}-\omega_{-}\right)^{2}}{\left(s-\omega_{-}\right)^{2}\left(\omega_{+}-t\right)^{2}}\right),
\end{gathered}
$$

as the worst cases for the singular terms involving $\partial_{\omega_{-}} \mathcal{R}_{0}, \partial_{\omega_{+}} \mathcal{R}_{0}, \partial_{\omega_{-}}^{2} \mathcal{R}_{0}, \partial_{\omega_{+}}^{2} \mathcal{R}_{0}$ and $\partial_{\omega_{-} \omega_{+}}^{2} \mathcal{R}_{0}$ respectively.

A.2. Regular entropy solutions. For the East type entropy case the integral representation (5.2) becomes

$$
\eta_{0}\left(\omega_{-}, \omega_{+}\right)=\int_{\omega_{-}^{*}}^{\omega_{-}}\left(\frac{\omega_{+}-\omega_{-}}{-t}\right)^{\frac{1}{2}} H\left(\frac{\omega_{+}\left(\omega_{-}-t\right)}{-t\left(\omega_{+}-\omega_{-}\right)}\right)\left(\theta_{-}^{\prime}(t)-\frac{1}{2 t} \theta_{-}(t)\right) d t .
$$

Thanks to the properties of $H$ and from representation (A.2), it is possible to give the following results on the regularity of $\eta_{0}$ near the umbilic point.

Proposition A.1. If $-\delta<\omega_{-} \leq \omega_{+}<\delta$, for a small positive constant $\delta$, and if

$$
\begin{aligned}
& \int_{\omega_{-}^{*}}^{-\delta}\left((-t)^{-\frac{3}{2}}\left(\theta_{-}^{\prime}(t)-\frac{1}{2 t} \theta_{-}(t)\right) d t=0\right. \\
& \int_{\omega_{-}^{*}}^{-\delta}\left((-t)^{-\frac{5}{2}}\left(\theta_{-}^{\prime}(t)-\frac{1}{2 t} \theta_{-}(t)\right) d t=0,\right.
\end{aligned}
$$

then

$$
\begin{gathered}
\eta_{0}=O\left(\left(\omega_{+}-\omega_{-}\right)^{\frac{1}{2}}\left(\omega_{+}^{2}\left(-\omega_{-}\right)+\omega_{+}\left(-\omega_{-}\right)^{2}\right)\right) \\
\frac{\partial \eta_{0}}{\partial \omega_{-}}=\frac{\partial \eta_{0}}{\partial \omega_{+}}=O\left(\omega_{+}^{\frac{3}{2}}\left(-\omega_{-}\right)+\omega_{+}\left(-\omega_{-}\right)^{\frac{3}{2}}\right) \\
\frac{\partial^{2} \eta_{0}}{\partial \omega_{-} \partial \omega_{+}}=\frac{\partial^{2} \eta_{0}}{\partial^{2} \omega_{-}}=\frac{\partial^{2} \eta_{0}}{\partial^{2} \omega_{+}}=O\left(\omega_{+}^{\frac{1}{2}}\left(-\omega_{-}\right)+\omega_{-}\left(-\omega_{+}\right)^{\frac{1}{2}}\right) .
\end{gathered}
$$

Proposition A.2. Suppose $\left(\eta_{0}, q_{0}\right)$ is an entropy-entropy flux pair, where $\eta_{0}$ is an entropy of type East or West with limit $\omega_{-}^{*}$. Under the same hypotheses as Proposition A.1, $\eta_{0}$ and $q_{0}$ have the following representations:

$$
\begin{aligned}
& \eta_{0}\left(\omega_{-}, \omega_{+}\right)=A_{0}\left(\omega_{-}, \omega_{+}\right) \theta_{-}\left(\omega_{-}\right)+\int_{\omega_{-}^{*}}^{\omega_{-}} B_{0}\left(x, \omega_{-}, \omega_{+}\right) \theta_{-}(x) d x, \\
& q_{0}\left(\omega_{-}, \omega_{+}\right)=C_{0}\left(\omega_{-}, \omega_{+}\right) \theta_{-}\left(\omega_{-}\right)+\int_{\omega_{-}^{*}}^{\omega_{-}} D_{0}\left(x, \omega_{-}, \omega_{+}\right) \theta_{-}(x) d x,
\end{aligned}
$$

where $A_{0}, B_{0}, C_{0}$ and $D_{0}$ are smooth functions in the variables $x, \omega_{-}, \omega_{+}$whenever $x<0$ and $\omega_{-}<0$. 


\section{REFERENCES}

[1] G.-Q. Chen and P.T. Kan, Hyperbolic conservation laws with umbilic degeneracy. I, Arch. Rational Mech. Anal. 130 (1995), no. 3, 231-276. MR.1337115 (96c:35111)

[2] _ Hyperbolic conservation laws with umbilic degeneracy, Arch. Ration. Mech. Anal. 160 (2001), no. 4, 325-354. MR1869669 (2003b:35130)

[3] K. N. Chueh, C. C. Conley, and J. A. Smoller, Positively invariant regions for systems of nonlinear diffusion equations, Indiana Univ. Math. J. 26 (1977), no. 2, 373-392. MR0430536 (55:541)

[4] R. Courant and D. Hilbert, Methods of mathematical physics. Vol. II, Wiley Classics Library, John Wiley \& Sons Inc., New York, 1989, Partial differential equations, Reprint of the 1962 original, A Wiley-Interscience Publication. MR.1013360 (90k:35001)

[5] R.J. DiPerna, Convergence of approximate solutions to conservation laws, Arch. Rational Mech. Anal. 82 (1983), no. 1, 27-70. MR684413 (84k:35091)

[6] _ Convergence of the viscosity method for isentropic gas dynamics, Comm. Math. Phys. 91 (1983), no. 1, 1-30. MR719807 (85i:35118)

[7] Compensated compactness and general systems of conservation laws, Trans. Amer. Math. Soc. 292 (1985), no. 2, 383-420. MR808729 (87g:35148)

[8] L.C. Evans, Partial differential equations, second ed., Graduate Studies in Mathematics, vol. 19, American Mathematical Society, Providence, RI, 2010. MR.2597943

[9] H. Frid and M.M. Santos, Nonstrictly hyperbolic systems of conservation laws of the conjugate type, Communications in partial differential equations 19 (1994), no. 1-2, 27-59. MR.1256997(95d:35100)

[10] X. Garaizar, The small anisotropy formulation of elastic deformation, Acta Appl. Math. 14 (1989), no. 3, 259-268. MR995288 (90j:73021)

[11] J. Glimm, The interaction of nonlinear hyperbolic waves, Comm. Pure Appl. Math. 41 (1988), no. 5, 569-590. MR948072 (89h:35198b)

[12] E.L. Isaacson, D. Marchesin, C.F. Palmeira, and B.J. Plohr, A global formalism for nonlinear waves in conservation laws, Comm. Math. Phys. 146 (1992), no. 3, 505-552. MR1167301(93h:35124)

[13] P.T. Kan, On the Cauchy problem of a $2 \times 2$ system of non-strictly hyperbolic conservation laws, Courant Institute of Math. Sciences, N.Y. University (1989), (Ph.D. Thesis, Advisor: J. Glimm).

[14] _ Non-strictly hyperbolic conservation laws, Appl. Math. Lett. 4 (1991), no. 4, 83-87. MR 1117777 (92c:35077)

[15] B.L. Keyfitz and H.C. Kranzer, A system of nonstrictly hyperbolic conservation laws arising in elasticity theory, Arch. Rational Mech. Anal. 72 (1979/80), no. 3, 219-241. MR549642 (80k:35050)

[16] P.D. Lax, The multiplicity of eigenvalues, Bull. Amer. Math. Soc. (N.S.) 6 (1982), no. 2, 213-214. MR640948 (83a:15009)

[17] Y. G. Lu, Convergence of the viscosity method for a non-strictly hyperbolic conservation law, Comm. Math. Phys. 150 (1992), no. 1, 59-64. MR1188496 (94k:35190)

[18] P. Marcati and A. Milani, The one-dimensional Darcy's law as the limit of a compressible Euler flow, J. Differential Equations 84 (1990), no. 1, 129-147. MR1042662 (91i:35156)

[19] P. Marcati and B. Rubino, Entropy methods for nonstrictly hyperbolic systems, J. Partial Differential Equations 10 (1997), no. 4, 333-346. MR.1486714 (99f:35135)

[20] F. Murat, Compacité par compensation, Ann. Scuola Norm. Sup. Pisa Cl. Sci. (4) 5 (1978), no. 3, 489-507. MR506997 (80h:46043a)

[21] B. Rubino, Approximate solutions to the Cauchy problem for a class of $2 \times 2$ nonstrictly hyperbolic systems of conservation laws, Boll. Un. Mat. Ital. B (7) 8 (1994), no. 3, 583-614. MR1294451 (95h:35135)

[22] Compactness framework and convergence of Lax-Friedrichs and Godunov schemes for a $2 \times 2$ nonstrictly hyperbolic system of conservation laws, Quart. Appl. Math. 53 (1995), no. 3, 401-421. MR 1343459 (96h:35122)

[23] D.G. Schaeffer and M. Shearer, The classification of $2 \times 2$ systems of nonstrictly hyperbolic conservation laws, with application to oil recovery, Comm. Pure Appl. Math. 40 (1987), no. 2, 141-178. MR872382(88a:35155)

[24] , Riemann problems for nonstrictly hyperbolic $2 \times 2$ systems of conservation laws, Trans. Amer. Math. Soc. 304 (1987), no. 1, 267-306. MR906816 (88m:35101)

[25] D. Serre, La compacité par compensation pour les systèmes hyperboliques non linéaires de deux équations à une dimension d'espace, J. Math. Pures Appl. (9) 65 (1986), no. 4, 423-468. MR881690 (88d:35123) 
[26] _ Systems of conservation laws. 2, Cambridge University Press, Cambridge, 2000, Geometric structures, oscillations, and initial-boundary value problems, Translated from the 1996 French original by I. N. Sneddon. MR1775057 (2001c:35146)

[27] M. Shearer, Loss of strict hyperbolicity of the Buckley-Leverett equations for three-phase flow in a porous medium, Numerical simulation in oil recovery (Minneapolis, Minn., 1986), IMA Vol. Math. Appl., vol. 11, Springer, New York, 1988, pp. 263-283. MR922970

[28] M. Shearer, D. G. Schaeffer, D. Marchesin, and P. L. Paes-Leme, Solution of the Riemann problem for a prototype $2 \times 2$ system of nonstrictly hyperbolic conservation laws, Arch. Rational Mech. Anal. 97 (1987), no. 4, 299-320. MR.865843 (88a:35156)

[29] J. Smoller, Shock waves and reaction-diffusion equations, Grundlehren der Mathematischen Wissenschaften [Fundamental Principles of Mathematical Science], vol. 258, Springer-Verlag, New York, 1983. MR688146 (84d:35002)

[30] Z. J. Tang and T. C. T. Ting, Wave curves for the Riemann problem of plane waves in isotropic elastic solids, Internat. J. Eng. Sci. 25 (1987), no. 11-12, 1343-1381. MR921358 (88m:73011)

[31] L. Tartar, Compensated compactness and applications to partial differential equations, Nonlinear analysis and mechanics: Heriot-Watt Symposium, Vol. IV, Res. Notes in Math., vol. 39, Pitman, Boston, Mass., 1979, pp. 136-212. MR584398 (81m:35014) 